\title{
REVIEW ARTICLE OPEN Endothelial activation and dysfunction in COVID-19: from basic mechanisms to potential therapeutic approaches
}

\author{
Yuefei Jin ${ }^{1}$, Wangquan $\mathrm{Ji}^{1}$, Haiyan $\mathrm{Yang}^{1}$, Shuaiyin Chen ${ }^{1}$, Weiguo Zhang ${ }^{1,2}$ and Guangcai Duan ${ }^{1}$
}

On 12 March 2020, the outbreak of coronavirus disease 2019 (COVID-19) was declared a pandemic by the World Health Organization. As of 4 August 2020, more than 18 million confirmed infections had been reported globally. Most patients have mild symptoms, but some patients develop respiratory failure which is the leading cause of death among COVID-19 patients. Endothelial cells with high levels of angiotensin-converting enzyme 2 expression are major participants and regulators of inflammatory reactions and coagulation. Accumulating evidence suggests that endothelial activation and dysfunction participate in COVID-19 pathogenesis by altering the integrity of vessel barrier, promoting pro-coagulative state, inducing endothelial inflammation, and even mediating leukocyte infiltration. This review describes the proposed cellular and molecular mechanisms of endothelial activation and dysfunction during COVID-19 emphasizing the principal mediators and therapeutic implications.

Signal Transduction and Targeted Therapy (2020)5:293

; https://doi.org/10.1038/s41392-020-00454-7

\section{INTRODUCTION}

In late 2019, an emerging viral pneumonia caused by a novel severe acute respiratory syndrome coronavirus 2 (SARS-CoV-2), termed coronavirus disease 2019 (COVID-19), was first reported in Wuhan, Hubei Province, China. ${ }^{1,2}$ This disease has infected more than 18 million people and lead to more than 500,000 deaths worldwide. $^{3}$ In general, COVID-19 is considered self-limited, and many of those infected have mild symptoms or appear to be asymptomatic; however, some patients develop respiratory failure which is the leading cause of death among infections with COVID$19 .^{4-6}$ To date, no specific drugs and vaccines are yet available for COVID-19, and its pathogenesis remains largely unclear.

Endothelial cells (ECs) mostly exist in the inner layer of all blood vessels and are normally protected by pericytes. ${ }^{7}$ Pulmonary ECs function as the basic barrier between blood and interstitium, accounting for one-third of cells in the lung. ${ }^{8,9}$ The activation and dysfunction of pulmonary ECs are considered signs of ARDS and the primary pathological causes. ${ }^{10,11}$ Accumulating evidence indicates that SARS-CoV-2 infection exerts adverse effects on the endothelium of capillary, which may contribute to COVID-19 pathogenesis by altering the integrity of vessel barrier, promoting pro-coagulative state, inducing endothelial inflammation, and even mediating leukocyte infiltration. ${ }^{12,13}$ Patients with severe or critical COVID-19 admitted in intensive care units frequently present underlying conditions (old age, diabetes, hypertension, and cardiovascular diseases). ${ }^{5}$ These comorbidities are often accompanied by years of chronic endothelial dysfunction. ${ }^{13,14}$ Endothelial activation and dysfunction are suggested to be related to the coagulation cascade. ${ }^{15}$ Established evidence suggests that activation of the coagulation pathway with the possible development of disseminated intravascular coagulation (DIC) is a feature of severe COVID-19 16,17 that may further result in thrombus formation. ${ }^{18}$ Therefore, it was suggested to name severe pulmonary COVID-19 as the microvascular COVID-19 lung vessels obstructive thromboinflammatory syndrome or MicroCLOTS. ${ }^{19}$

To date, the ongoing global pandemic of COVID-19 still poses a considerable threat to many people. ${ }^{20}$ An improved mechanistic understanding of endothelial activation and dysfunction is of utmost importance. This review describes the possible cellular and molecular mechanisms of endothelial activation and dysfunction in COVID-19, emphasizing the principal mediators and the therapeutic implications.

\section{PATHOPHYSIOLOGY OF ECS}

The primary histological characteristic of resting ECs is their cobblestone shape; however, they constitute more than static mechano-protective plates (Fig. 1).21 ECs mostly exist in the inner layer of blood vessels and are normally protected by pericytes that support the vessel structure. ${ }^{7}$ ECs have different functions and structures depending on the tissues and organs. For example, pulmonary vascular ECs are arranged in a dense monolayer, forming a protective barrier that is in direct contact with blood components. ${ }^{9}$ The commonly accepted functions of ECs in the homeostasis of body physiology are controlling vascular permeability and regulating vascular tone (Fig. 1). ${ }^{21}$ ECs can synthesize and release various endothelium-derived relaxation factors, such as nitric oxide (NO) and prostaglandin (PG), and contractile factors, including endothelin (ET), thromboxane A2 (TXA2), reactive oxygen species (ROS), and angiotensin II (Ang II), which play significant roles in the regulation of vascular tone (Fig. 1). ${ }^{22}$ When activated, ECs secrete chemoattractants, cytokines, and adhesion molecules, leading to augmented blood vessel permeability (Fig. 1). ${ }^{21}$ In resting ECs, the synthesis of these molecules can be suppressed by NO. ${ }^{23}$ In addition, ECs are also involved in adhesion and aggregation of platelets, activation, adhesion, and migration

\footnotetext{
${ }^{1}$ Department of Epidemiology, College of Public Health, Zhengzhou University, Zhengzhou 450001, People's Republic of China and ${ }^{2}$ Department of Immunology, Duke University Medical Center, Durham, NC 27710, USA

Correspondence: Guangcai Duan (gcduan@zzu.edu.cn)
}

Received: 10 August 2020 Revised: 6 October 2020 Accepted: 23 November 2020

Published online: 24 December 2020 

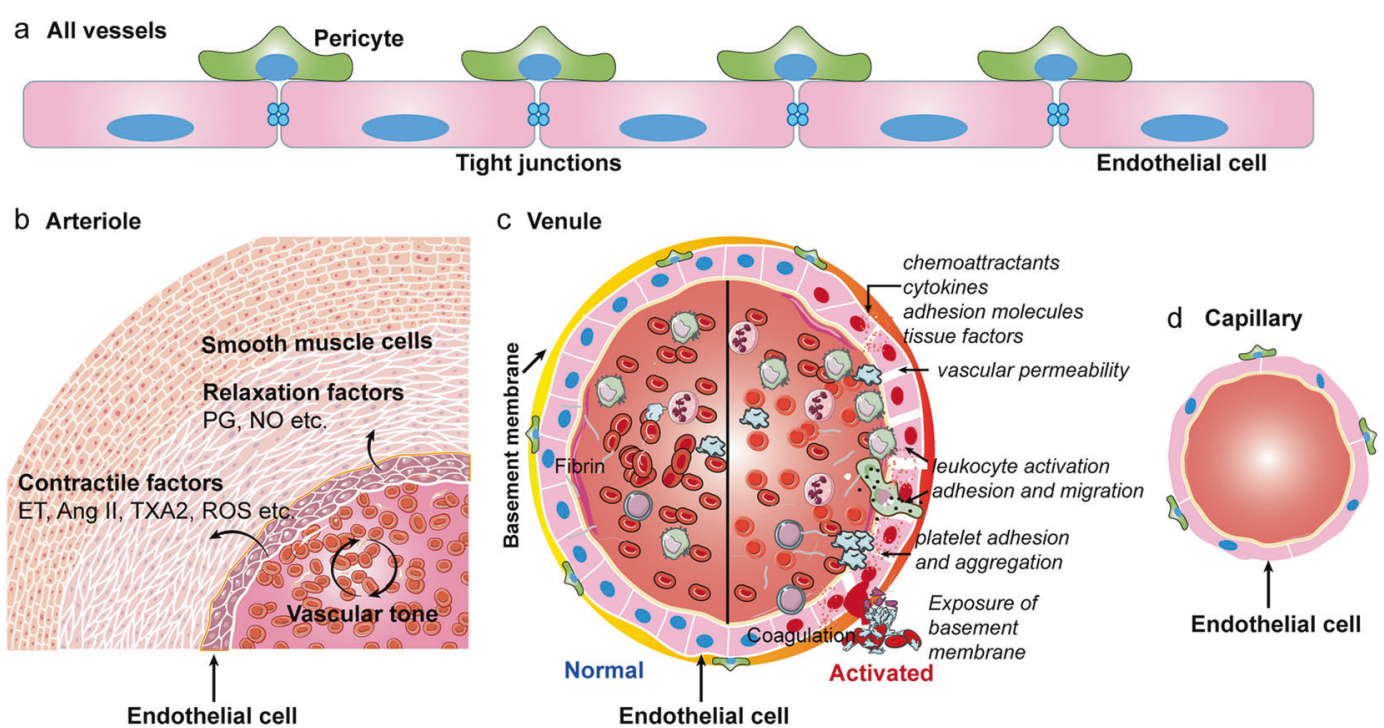

Fig. 1 Pathophysiology of endothelial cells. Endothelial cells exist in the inner layer of blood vessels (a) such as arteries (b), veins (c), and capillaries (d), and are normally protected by pericytes that support the vessel structure. Tight junctions link neighboring cells and help maintain tissue integrity, act as barriers to permeability. The regulation of vascular tone, and permeability upon activation are illustrated

of leukocytes, and fibrin balance (Fig. 1). ${ }^{24} \mathrm{NO}$ exerts direct effects on leukocytes, preventing their activation into motile forms that are capable of entering tissues. ${ }^{23}$ However, dysfunctional endothelial response to damage or infection cannot produce sufficient amounts of NO. ${ }^{23}$ Therefore, a decline in NO bioavailability always represents endothelial dysfunction.

\section{ENDOTHELIAL ACTIVATION AND DYSFUNCTION ARE ASSOCIATED WITH COVID-19 SEVERITY}

Epidemiological studies suggest that severe cases or deaths due to COVID-19 frequently present with underlying comorbidities, such as advanced age, hypertension, diabetes, and cardiovascular diseases. $^{5}$ Chronic vascular endothelial injury often co-occurs in patients with such comorbidities. ${ }^{14}$ Most patients with critical COVID-19 die from ARDS, pulmonary edema, cytokine storm, multiple organ failure, and diffuse coagulopathy. ${ }^{4}$ Among the aforementioned causes, ARDS has been considered a result of pulmonary EC damage. ${ }^{12}$ The direct or indirect activation of ECs mediates the extensive production of inflammatory cytokines, adhesion molecules, and chemokines, which may result in cytokine storm, local inflammatory cell infiltration, and vascular leakage. ${ }^{12,21,23}$ The plasma levels of adhesion molecules, such as intercellular adhesion molecule 1 (ICAM-1), fractalkine, vascular cell adhesion molecule-1 (VCAM-1), vascular adhesion protein-1 (VAP-1), and vascular endothelial growth factor (VEGF), had been reported to be elevated among COVID-19 patients, especially in severe patients. ${ }^{25,26}$ Clinical findings also indicate an increased occurrence of Kawasaki disease, a form of vasculitis, in pediatric patients with COVID-19, implying acute vascular inflammation. ${ }^{27,28}$

The dysfunction of ECs fails to release sufficient amounts of NO, resulting in vessel constriction. ${ }^{23} \mathrm{NO}$ deficiency has been observed among COVID-19 patients, ${ }^{29}$ and it may cause vascular smooth muscle contraction, reducing the ability to neutralize ROS and NOmediated antiviral capability. ${ }^{30,31}$ ECs function as key regulators of coagulation and as counterbalance for thrombin. ${ }^{32} \mathrm{~A}$ dysfunctional endothelial response to viral infection can activate the coagulation pathway and lead to anticoagulation imbalance. ${ }^{7}$ Comprehensive coagulation analyzes of patients with COVID-19 indicate elevated levels of D-dimer, increased fibrinogen, enhanced platelet activation, and increased variables of viscoelastic in the plasma of severe cases. ${ }^{33,34}$ Elevated D-dimer levels in critical patients represent poor prognosis. ${ }^{35}$ In addition to venous thromboembolism, the association of microthrombus formation with multiple organ failure and acro-ischemic change has been proposed. $^{36}$ The pathological manifestations leading to severe COVID-19 have been recently considered as vascular leakage, inflammatory reactions, anticoagulation imbalance, and endothelial dysfunction, which may play a central role in the aforementioned procedure. ${ }^{12}$ Therefore, understanding the mechanisms of endothelial activation and dysfunction during the course of COVID-19 infection will help in the early identification of individuals which are at risk of suffering from severe complications, and thus, provide appropriate therapeutic targets. The overview of endothelial activation and dysfunction in COVID-19 pathogenesis is shown in Fig. 2.

\section{CRITICAL FUNCTIONS OF ECS IN COVID-19 INFLAMMATION}

Innate immune receptor-mediated inflammatory responses in ECs EC functions can be regarded from two aspects in the immunology of vascular homeostasis and pathology. First, ECs are a constitutive and integral part of the vascular system, and thus intrinsically cause vascular diseases once they are dysfunctional. Second, ECs actively mediate inflammatory or immune responses at infection or injury sites. ${ }^{21,22}$ Innate immunity serves as the first line of defense against microbial pathogens. It depends on a limited number of functional proteins or pattern recognition receptors (PRRs), such as Toll-like receptors (TLRs), nucleotidebinding oligomerization domain (NOD)-like receptors (NLRs), or retinoic acid-inducible gene I (RIG-l)-like receptors (RLRs). ${ }^{37}$ Inflammation is commonly triggered when PRRs detect tissue damage or microbial infection. ${ }^{37,38}$ To date, our knowledge of specific innate immune responses to SARS-CoV-2 in ECs remains unknown. However, the virus-host interactions involving SARSCoV-2 are likely to recapitulate many of those involving other virus types or microbial infections given the conserved mechanisms of innate immunity. PRR-mediated detection of viral single-stranded RNA (ssRNA) and double-stranded RNA (dsRNA), termed pathogen-associated molecular patterns (PAMPs), and further activation of antiviral innate immune response have been observed during SARS-CoV infection. ${ }^{39,40}$ Existing pharmacological modulators of PRRs mediated inflammatory responses are listed in Table 1. 


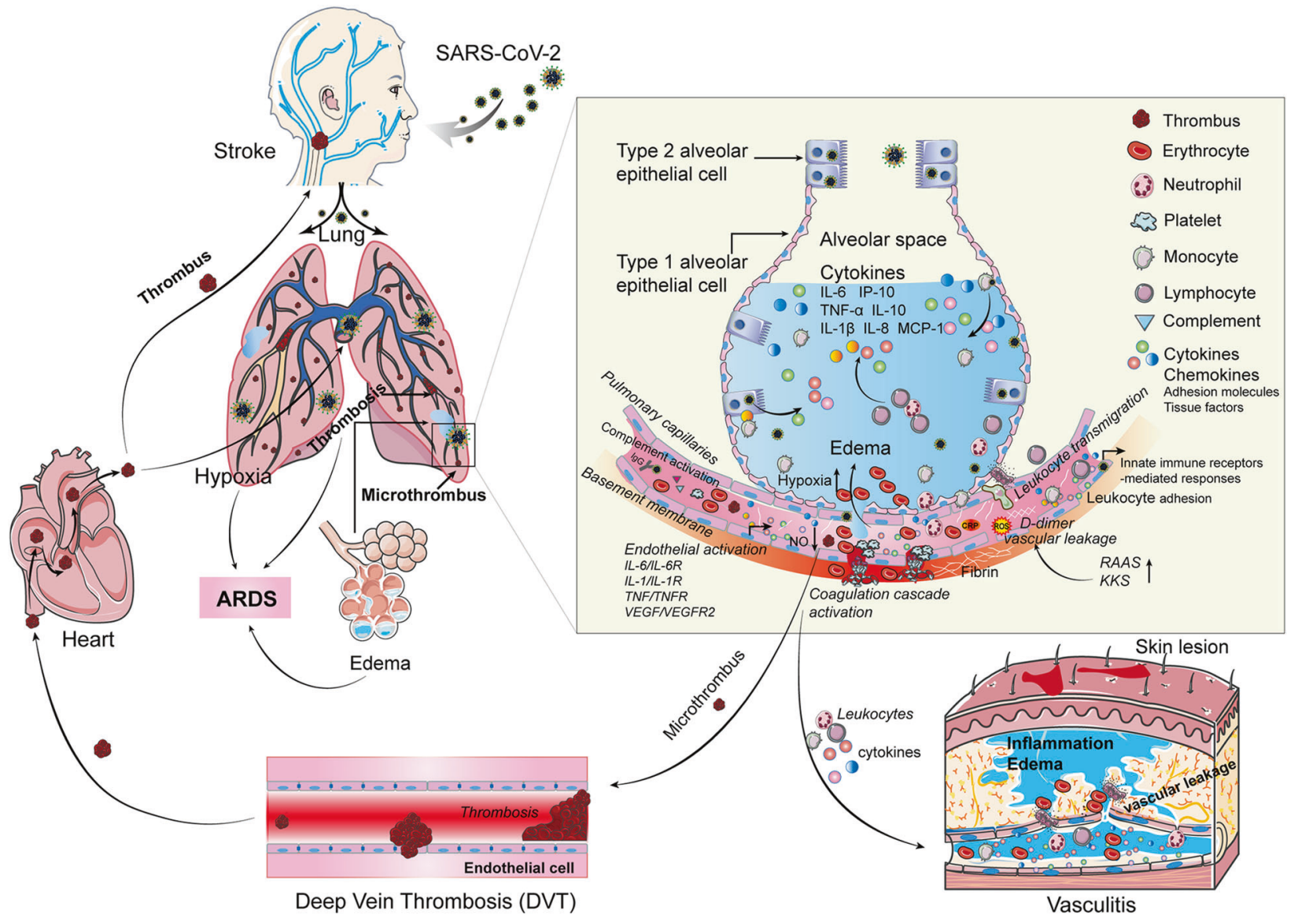

Fig. 2 Overview of endothelial activation and dysfunction in the pathogenesis of COVID-19. In the initial stage of severe COVID-19 patients, SARS-CoV-2 infection causes acute lung injury, and then excessive cytokines are released from immune cells, bronchial epithelial cells, and alveolar cells. SARS-CoV-2 infection and various cytokines are predicted to cause endothelial activation and dysfunction by multiple pathways, leading to vascular inflammation and permeability. Then more immune cells enter or migrate into alveoli and enhance lung inflammation. With vascular permeability, erythrocytes enter into alveoli, leading to edema. Moreover, with the release of pro-inflammatory cytokines and inflammatory cells to circulation, vasculitis occurs. The disruption of vascular integrity and EC apoptosis leads to the exposure of the thrombogenic basement membrane and the activation of the clotting cascade. Endothelial cells release relevant cytokines that further augment platelet production. Platelet activation is the primary cause of thrombosis. Inflammation, edema, and microthrombus work together to cause ARDS. The transfer of microthrombi into the blood circulation increases the risk of the formation of deep vein thrombosis, which may further cause pulmonary embolism and stroke

TLRs. TLR1 6 and TLR9 have been detected in all types of tissuespecific ECs. ${ }^{41,42}$ Poly (I:C), a synthetic dsRNA analog, can directly activate TLR1/2, TLR3, and TLR4. ${ }^{41}$ Upon activation through TLR, nuclear factor kappa-B (NF-kB) and mitogen-activated protein kinase (MAPK) signaling are initiated via myeloid differentiation factor 88 (MyD88) and/or TIR-domain-containing adapter-inducing interferon- $\beta$ (TRIF). ${ }^{41}$ This process elicits a hyper-inflammatory response by promoting the production of interleukin (IL)-6, IL-8, tumor necrosis factor (TNF)- $\alpha$, and IL-1 $\beta$, and adhesion molecules (E-selectin, P-selectin, ICAM, and VCAM). Consequently, vascular permeability is elevated by disrupted junction protein claudin- 5 and induction of procoagulant factors, such as tissue factors, plasminogen activator inhibitor type-1 (PAI-1), von Willebrand factor (vWF), and the urokinase plasminogen activator (uPa). ${ }^{42}$ While TLR7/8 is not detected in ECS, human umbilical vein endothelial cells (HUVECs) express TLR3. ${ }^{43} \mathrm{ECs}$ also produce type I interferons, such as IFN-a. IFN-a is well known as a significant cytokine during antiviral responses. ${ }^{43}$ Moreover, TLR9 in ECs can be activated when DAMPs, such as DNA and proteins released outside the cell following tissue injury, are recognized. ${ }^{44}$ Once activated, rapid phosphorylation of NF-KB and adhesion molecules E-selectin and ICAM-1-independent MAPK signaling is detected. ${ }^{44}$
In addition, sepsis-related lung inflammation appears to be dependent on the activation of endothelial TLR4-regulating neutrophil sequestration into the lungs. ${ }^{45}$ Collectively, TLRs in ECs are not only important in host defense against infections but they also contribute to microvascular dysfunction and inflammation during systemic infections.

NLRs. The NOD proteins NOD1 and NOD2 are two members of NLR family, which function as cytoplasmic PRRs. ${ }^{41}$ Different types of ECs, such as HUVECs, microvascular ECs and human aortic endothelial cells, express NOD 1. ${ }^{41,46}$ Upon microbial stimulation, ECs had been shown to produce IL-8 with a NOD1-dependent manner. ${ }^{46,47}$ The NLR proteins containing a PYD (NLRPs) are classified as another NLR subgroup. NLRPs interact with ASC and caspase-1, forming multiprotein complexes called inflammasomes. ${ }^{48}$ Inflammasomes (e.g., NLRP1 and NLRP3) regulate the proteolytic processing of prolL-1 $\beta$ and prolL-18 into mature forms, and an inflammatory cell death termed pyroptosis. ${ }^{41}$ NLRP1/3, ASC, and caspase- 1 expression have been confirmed in lung and vascular ECs. ${ }^{49,50}$ The NLRP3 pathway has been recently considered as a novel target for treatment of COVID-19. 
Table 1. Summary of existing pharmacological modulators that act directly or indirectly on innate immune receptors-mediated inflammatory responses in endothelial cells

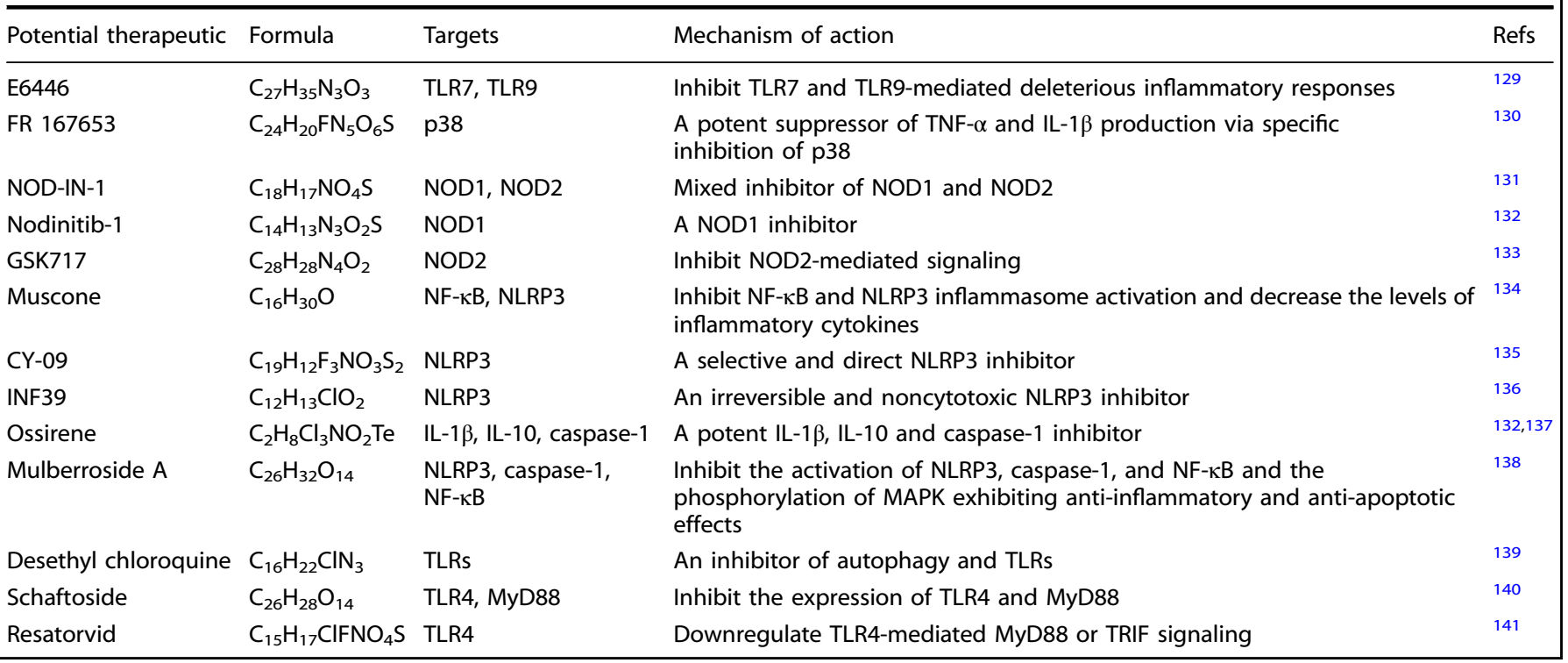

RLRs. RIG-I and melanoma differentiation-associated gene 5 (MDA5) are two important cytoplasmic sensors for viral RNA, which belong to the RLRs family of PRR. ${ }^{37,41,42}$ RIG-I and MDA5 have been detected ECs (e.g., HUVECs), and RIG-I expression is upregulated upon microbial stimulation. ${ }^{37,41}$ The CARD-containing adapter molecule mitochondrial antiviral-signaling protein (MAVS) is engaged by both RIG-I and MDA5, which in turn stimulate the signaling pathways resulting in IRF3/7-dependent IFN- $\alpha / \beta$ response and NF-KB-dependent inflammatory genes transcription. ${ }^{37}$

ECs in adaptive immune responses

Peptide-major histocompatibility complexes (MHCs) and costimulatory molecules are generally required for T cell activation. ${ }^{52}$ All blood vessels are lined by ECs forming a barrier between blood immune cells and parenchymal tissues, and the interaction between $T$ cells and organ resident ECs has mostly remained elusive. Interestingly, a recent study using single-cell transcriptomics found that high levels of genes related to MHC class II-mediated antigen presentation were detected in a subtype of lung capillary ECs, ${ }^{53}$ suggesting a potential role as antigen-presenting cells (APCs) to function in immune surveillance against respiratory pathogens for this EC subtype. However, given the lack of CD80/CD86 costimulatory molecules on the cell surface, ECs are not able to activate naïve T cells. Thus, they probably function as semi-professional APCs. ${ }^{54}$ When reactivated by IFN- $\gamma$ (mostly derived from Th1 cells) to express MHC molecules, ECs effectively stimulate cytokine production and proliferation of CD4/8 memory $\mathrm{T}$ cells. ${ }^{55}$ It was reported that microvascular ECs could stimulate the transendothelial migration of effector memory CD4 T cells. ${ }^{23}$ Additionally, the ECs from chronically inflamed tissues may function in the polarization of inflammatory responses related to adaptive immunity. ${ }^{23}$ Overactivation of $\mathrm{T}$ cells, characterized by increased numbers of Th17 cells and hyperactivation of CD8 T cells, had been detected in the peripheral blood of infections with COVID$19 .^{56}$ Taken together, above evidence indicates that the interaction of ECs with T cells may lead to excessive inflammation in severe infections with COVID-19.

Endothelial adhesion molecule-dependent leukocyte recruitment Venular ECs form the primary site of leukocyte trafficking from the circulating blood into the tissues. ECs are assumed to participate in leukocytes recruitment from the bloodstream into the sites of infection and inflammation. ${ }^{23}$ During the process of SARS-CoV-2 infection, inflammatory cytokines of IL-1 and TNF-a derived from activated leukocytes, bind to the extracellular domains of IL-1 receptor 1 (IL-1R1) and TNF receptor 1 (TNFR1) on the surface of the endothelial membrane, further initiating various kinase cascades and leading to the activation of NF-KB and activator protein 1 (AP1). ${ }^{23}$ These transcription factors induce adhesion molecules (ICAM-1, VCAM-1, E-selectin, and P-selectin). VCAM-1 was originally defined as a CD11-/CD18-independent endothelial ligand for mononuclear leukocytes. $^{23}$ It recognizes $\alpha 4 \beta 1$ and $a 4 \beta 7$ integrins of leukocyte. VCAM- 1 is recently emerged as a key inducible EC-expressed adhesion molecule that mediates the recruitment of monocyte to injury and infection sites. ${ }^{23}$ ICAM-1, expressed on the surface of endothelium and in the peripheral vasculature, is upregulated in lesions. Through binding with leukocyte $\beta 2$ integrins (CD11/CD18), ICAM-1 supports leukocyte arrest and firm adhesion and mediates the transmigration of monocytes and lymphocytes. ${ }^{23}$ Neutrophils and $T$ cells, particularly regulatory $T$ cells (Tregs) in the peripheral blood of humans, possess E-selectin ligands, and are thus recruited. ${ }^{23,57}$ P-selectin is an adhesion molecule, and mostly expressed on platelets and endothelium, promoting rolling, adhesion, and transmigration of leukocytes by binding to a disulfide-bonded homodimeric mucin-like glycoprotein, P-selectin glycoprotein ligand-1 (PSGL-1) expressed by leukocytes. ${ }^{58}$ P-selectin plays an important role in leukocyte-endothelial interactions, particularly in modulating inflammatory pathways and defense against infections. ${ }^{58}$ As mentioned earlier, the serum levels of adhesion molecules (ICAM-1, VCAM-1, E-selectin, and P-selectin) in severe infections with COVID-19 are significantly increased. ${ }^{25,59}$ Autopsy biopsy of SARS-CoV-2-infected lungs exhibits mononuclear and polymorphonuclear aggregation, accompanied by the apoptotic ECs. ${ }^{60}$ We speculate that these adhesion molecules expressed by ECs mediate inflammatory cell infiltration and EC injury caused by leukocytes contributes to inflammation, particularly in the capillaries during COVID-19 progression. Existing pharmacological modulators that act directly or indirectly on endothelial activation-mediated leukocytes recruitment are listed in Table 2.

\section{ENDOTHELIAL ACTIVATION AND DYSFUNCTION ARE} ASSOCIATED WITH THROMBOSIS FORMATION DURING COVID-19 Increasing evidence worldwide suggests that patients with severe COVID-19 frequently develop pulmonary embolism (PE), deep vein 
Endothelial activation and dysfunction in COVID-19: from basic mechanisms... Jin et al.

Table 2. Summary of existing pharmacological modulators that act directly or indirectly on endothelial activation-mediated leukocytes recruitment

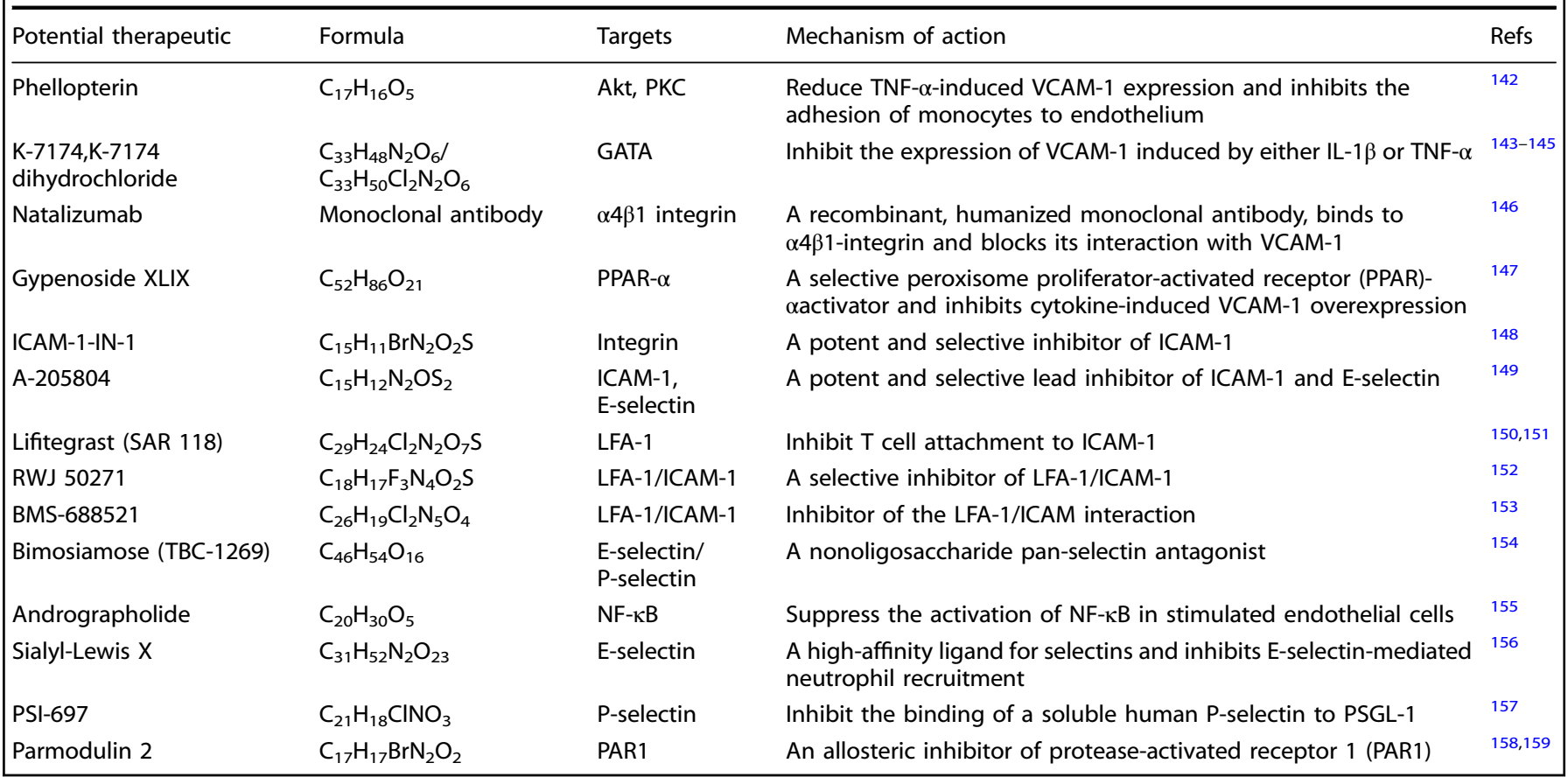

thrombosis (DVT), stroke, and even thrombosis in the extracorporeal circuits and arterial thrombosis (Fig. 2). ${ }^{12,61-63} \mathrm{EC}$ swelling with foamy degeneration and a few areas of segmental fibrin thrombus in glomerular capillary loops were found in patients who have died from COVID-19 likely due to excessive endothelial activation and dysfunction. ${ }^{60}$ The association of microthrombus formation with organ dysfunction and ARDS has been proposed recently. ${ }^{12,34}$ Coagulation pathway activation with the potential development of DIC is a general characteristic of severe infections with COVID-19, and one of the most common findings is the increase of fibrin degradation fragments (D-dimer). ${ }^{12,33,64}$ Coagulation is a highly well-organized procedure that involves the interaction of ECs, platelets, and coagulation factors. ${ }^{32}$ Upon endothelial activation and dysfunction, disruption of vascular integrity and EC apoptosis results in exposure of the thrombogenic basement membrane and activation of the clotting cascade. $^{7}$ In addition, ECs activated by IL-1 $\beta$ and TNF- $\alpha$ can trigger coagulation by displaying vWF, P-selectin, and fibrinogen, onto which platelets bind. ${ }^{23}$ In turn, ECs release relevant cytokines which augment platelet production. Platelet activation is the primary cause of thrombosis. ${ }^{15}$ Platelets also produce VEGF, which promotes ECs to express the tissue factor, i.e., the main activator of the coagulation cascade. ${ }^{15}$ In response, the fibrinolytic system is activated and releases D-dimers into the circulation. ${ }^{36}$ ARDS develops due to the DIC and clogging of capillaries by inflammatory leukocytes and possible thrombosis in larger blood vessels. To date, at least three strategies, namely, heparin for VTE prevention, anticoagulant, and anti-platelet therapies, have been suggested to treat coagulation abnormalities and thrombosis related to endothelial activation and dysfunction (Table 3). ${ }^{36}$

\section{PROPOSED MECHANISMS FOR ENDOTHELIAL ACTIVATION AND DYSFUNCTION IN COVID-19}

SARS-CoV-2 infection directly induces EC apoptosis

Established evidence suggests that SARS-CoV-2 hijacks the cell membrane receptor ACE2 to invade host cells with involvement of transmembrane protease serine 2 (TMPRSS2). Human ECs express ACE2 and TMPRSS2 and are considered SARS-CoV-2 target cells. ${ }^{1,65}$ SARS-CoV-2 replication is detected in ECs from various organs of patients with COVID-19 or engineered human blood vessel organoids. ${ }^{60,66-68}$ Autopsy pathology shows the presence of the virus and rupture of the cell membrane of pulmonary ECs. ${ }^{63}$ Moreover, SARS-CoV-2 proliferation in ECs directly induces damage and apoptosis (Fig. 3). ${ }^{60}$ We reviewed several antiviral drugs in our previous publication. ${ }^{4}$ At present, several monoclonal neutralizing antibodies against SARS-CoV-2 have been developed to treat COVID-19 patients. $^{69,70}$

Loss of ACE2 leads to the imbalance of the renin-angiotensin-aldosterone system (RAAS) and the kallikrein-kinin system (KKS)

ACE2 not only is the receptor for viral entry but also an important component of RAAS. ${ }^{71}$ ACE2 plays a significant role in self-repair of $\mathrm{ECs}^{71}$ and the development of acute lung failure caused by SARS$\mathrm{CoV}$, and other viruses (e.g. avian influenza A strains) by modulating RAAS. ${ }^{72}$ The amount of ACE2 in ECs is decreased as a result of competitive binding and shedding induced by TNF-a and metalloprotease 17 (ADAM17) ${ }^{73,74}$ Consequently, ACE2 fails to catalyze the conversion of Ang II to Ang (1-7), leading to accumulation of Ang II and pathological damage. ${ }^{75}$ Clinical reports have suggested that the level of Ang II in COVID-19 patients is considerably higher than that in healthy individuals. ${ }^{76}$ Ang II is an autocrine vasoconstrictor of ECs. ${ }^{77}$ Excessive Ang II activates the PI3K-Akt signaling pathway through the AT1 receptor to regulate endothelial activation and production of IL-6 and ROS (Fig. 3). ${ }^{78-81}$ In addition, high concentrations of Ang II cause EC death and vascular degeneration by destroying the connection between ECs and pericytes. ${ }^{82,83}$ KKS has been suggested to regulate many physiological processes, such as inflammation, coagulation, vasodilation, and blood pressure. ECs are assumed to be a target of KKS. $^{84,85}$ Under inflammation or infection, the expression of the bradykinin receptors (B1R and B2R) of KKS is upregulated in ECs. However, a decrease in the quantity and activity of ACE2 fails to inactivate the ligands of B1R, Lys des-Arg9-BK, and des-Arg9-BK in the lungs, and in turn, activates KKS (Fig. 3). ${ }^{86,87}$ Activated KKS will cause endothelial dysfunction and further result in leukocyte adhesion, complementing activation. ${ }^{8,89}$ We have summarized 
Table 3. Potential therapeutic tools for anticoagulant and antithrombotic treatment in COVID-19

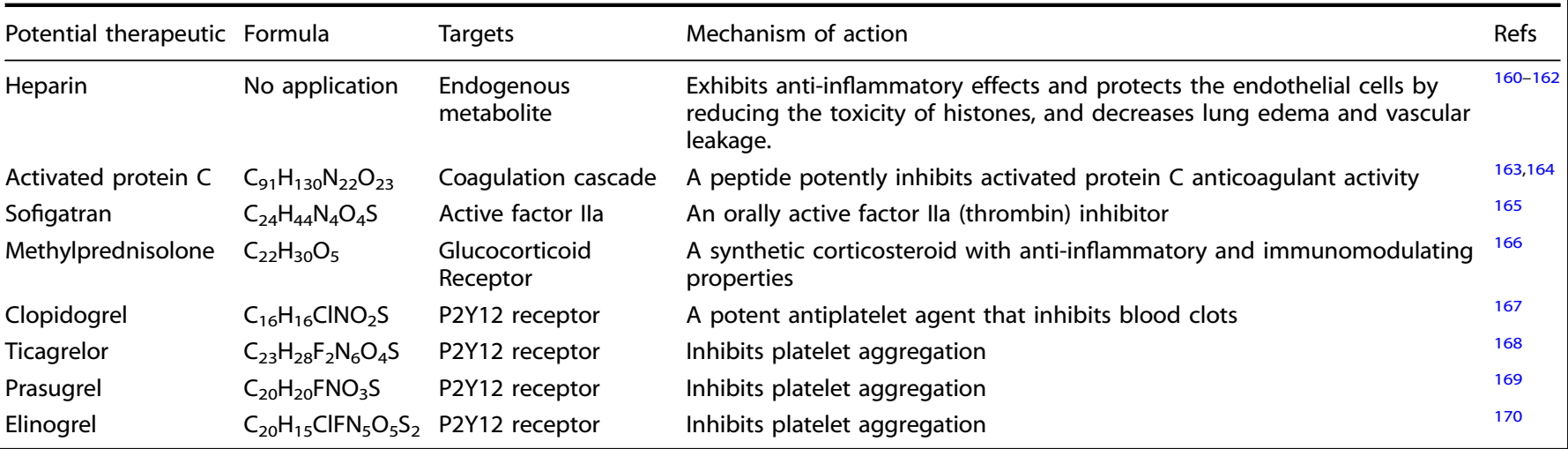

potential therapeutic tools for suppressing RAAS and KKS activation (Table 4), except for RAAS inhibitors, including ACE inhibitors and angiotensin II receptor blockers (ARBs), which have been indicated no benefit for COVID-19 protection. ${ }^{65}$

HMGB1-RAGE/TLR4 signaling-mediated endothelial activation and dysfunction

The high mobility group box 1 (HMGB1), a ubiquitous nuclear protein has been demonstrated to cause endothelial activation and dysfunction. ${ }^{90,91}$ During the process of hypoxic cellular stress or necrosis, HMGB1 is released into the extracellular environment and becomes a lethal pro-inflammatory mediator due to engagement of the receptor for advanced glycation products (RAGE) and TLR4. ${ }^{92,93}$ Upon RAGE or TLR4 activation, adhesion molecules (e.g., ICAM-1, P-selectin, and VCAM-1) and pro-inflammatory cytokines (TNF-a, IL-1, and IL-6) are induced through activating Src kinase, MAPK, and NF-KB. ${ }^{94-96}$ HMGB1 can increase the permeability of endothelial cell monolayer via the RAGE-Src pathway (Fig. 3). ${ }^{95}$ HMGB1 has been considered a potential marker of acute lung injury complicated by ARDS, notably viral pneumonia, such as SARS. ${ }^{97-99}$ Therefore, an increase in HMGB1 is predicted during the progress of COVID-19. At present, HMGB1 has been declared as a therapeutic target for treatment of COVID-19. ${ }^{100}$ The agents that can inhibit HMGB1-RAGE/TLR4 signaling are listed in Table 5.

Oxidative stress- and ROS formation-mediated endothelial dysfunction

Oxidative stress is defined as a state of the excessive generation of oxidant compounds and/or the reduction in savaging antioxidants. $^{101}$ Its sequelae include elevated levels of oxidized biomolecules and related tissue damage. Increasing evidence indicates that oxidative stress plays an important role in promoting endothelial dysfunction. ${ }^{101}$ A number of mechanisms may participate in oxidative stress-mediated endothelial dysfunction, but the predominant mechanism is likely to associate with reduced NO bioavailability. ${ }^{101}$ Decreased endothelial nitric oxide synthase (eNOS) expression, lack of substrates for eNOS, eNOS inactivation, and accelerated NO degradation have been considered to cause a decline in NO bioavailability. ${ }^{101}$ As mentioned earlier, the serum level of NO among patients with COVID-19 is decreased, implying oxidative stress. ${ }^{29}$ In general, another main source of oxidative stress is ROS derived from mitochondria. ${ }^{101}$ Physiological ROS generation is required for maintaining the regular vascular homeostasis. ${ }^{101}$ In the vasculature, some enzyme systems, such as NADPH oxidase (NOX) and differentially localized and expressed eNOS, participate in ROS formation. Among these systems, NOX apparently plays a crucial role in orchestrating the activation and dysfunction of other enzymes. This process is considered the major source of ROS in the vascular endothelium. ${ }^{102}$ Acute inflammation by multiple mechanisms contributes to COVID-19 pathogenesis. ${ }^{40}$ Under acute inflammation status, however, excess ROS production can cause oxidation of macromolecules, promoting cell apoptosis mediated by cytochrome-c release. ${ }^{101}$ ROS is also capable of activating calcium signaling and NF-KB signaling to induce adhesion molecules and proinflammatory cytokines, which can increase vascular permeability and promote leukocyte adhesion (Fig. 3). ${ }^{23,103,104} \mathrm{~A}$ recent study suggests that oxidative stress caused by NOX2 activation contributes to COVID-19 pathogenesis and is associated with thrombotic events in COVID-19 patients. ${ }^{105}$ Therefore, a beneficial effect of antioxidant drugs (Table 6) on endothelial function should be considered for the treatment of COVID-19 in the future.

IL-6-/IL-6R-mediated endothelial activation and dysfunction Clinical reports have suggested that an increasing level of circulating IL- 6 is related to the pathogenesis of COVID-19., 106 IL- 6 is produced by multiple cell types that include monocytes/ macrophages, adipocytes, and ECs and is elevated in circulation during inflammatory conditions. ${ }^{107}$ Through the engagement of IL-6 receptor (IL-6R), IL-6 initiates the JAK-STAT pathway ${ }^{80}$ and in turn upregulates adhesion molecules, (VCAM-1, ICAM-1, Eselectin), and MCP-1, enhancing leukocyte adherence and extravasation into the vascular wall. ${ }^{80}$ In addition to classical IL$6 \mathrm{R}$ signaling, IL- 6 is known to reduce NO bioavailability and increase oxidative stress, leading to endothelial permeability, along with the recruitment and infiltration of the vascular wall by circulating leukocytes (Fig. 3). ${ }^{80}$ Potential therapeutic approaches for targeting IL-6/IL-6R signaling are listed in Table 7.

Complement activation contributes to endothelial activation and dysfunction

Complement $(C)$ is an essential part of the innate immunity that serves as a first line of defense against microorganisms. ${ }^{108}$ Complement system comprises of over 30 components, containing membrane-bound regulators, receptors, and numerous plasma proteins. It is well known that complement can be activated through the classical, lectin, or alternative pathways. ${ }^{109}$ The role of complement in the pathogenesis of COVID-19 is to attract more attention. ${ }^{110}$ The complement activation has been confirmed in the pathogenesis of COVID-19, and excessive complement activation leads to acute and chronic inflammation, endothelial dysfunction, and thrombus formation. ${ }^{111,112}$ Activation and amplification of complement generates various potent effectors. Among these effectors, $\mathrm{C} 5$ a serves as the dominant effector in signaling danger, and the induction of immune modulatory responses. ${ }^{109}$ As the strongest anaphylatoxin, C5a can recruit neutrophils and other leukocytes to the site of activation and prime them through binding to $\mathrm{C} 5 \mathrm{a}$ receptor 1 (C5aR1), promoting VE-cadherin degradation. VE-cadherin degradation further results in disruption of the endothelial 


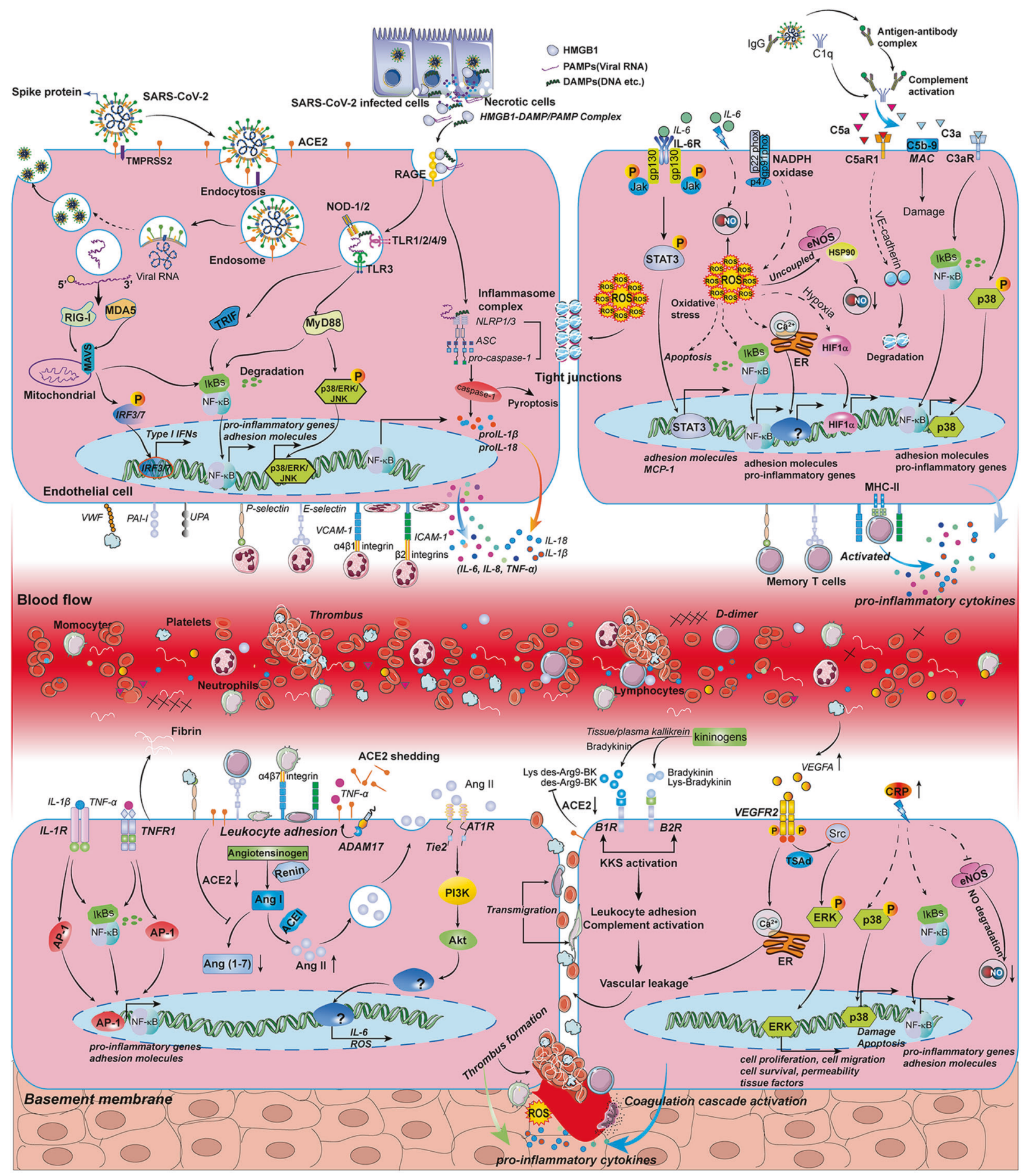

Fig. 3 Proposed mechanisms of endothelial activation and dysfunction during COVID-19 This picture highlights possible mechanisms of endothelial activation and dysfunction during SARS-CoV-2 infection, including loss of vascular integrity, vascular permeability, activation of the coagulation pathway, inflammation, and thrombus formation

barrier. ${ }^{108,109,113}$ The membrane attack complex (MAC/C5b-9) of complement has also been reported to play an important role in endothelial dysfunction during immune complex vasculitis. ${ }^{114}$ In addition to $\mathrm{C} 5 \mathrm{a}$ and $\mathrm{MAC} / \mathrm{C} 5 \mathrm{~b}-9, \mathrm{C} 3 \mathrm{a}$ has been demonstrated to upregulate adhesion molecules (ICAM-1 and VCAM-1) in ECs through p38 MAPK and NF-KB activation (Fig. 3). A recent report indicates that the plasma levels of sC5b-9 and C5a are elevated in COVID-19 patients, and complement activation has been suggested as a novel therapeutic target. ${ }^{112,115}$ Relative agents that can suppress complement activation are listed in Table 8.

VEGFA/VEGFR2 signaling-mediated endothelial activation and dysfunction

VEGFs, secreted by a range of cells, are well known for their participation in orchestrating the development and maintenance of blood vascular systems. ${ }^{116}$ They bind to their cognate tyrosine 
Table 4. Summary of existing pharmacological modulators that act directly or indirectly on RAAS and KKS activation during COVID-19

\begin{tabular}{lllll}
\hline Potential therapeutic & Formula & Targets & Mechanism of action \\
\hline Resorcinolnaphthalein & $\mathrm{C}_{24} \mathrm{H}_{14} \mathrm{O}_{5}$ & ACE2 & A specific ACE2 enhancer \\
SL910102 & $\mathrm{C}_{30} \mathrm{H}_{30} \mathrm{~N}_{6} \mathrm{O}$ & AT1 receptor & A unlabeled nonpeptide AT1 receptor antagonist \\
BMS-248360 & $\mathrm{C}_{36} \mathrm{H}_{45} \mathrm{~N}_{5} \mathrm{O}_{5} \mathrm{~S}$ & AT1 receptor & An antagonist of AT1 receptor \\
Losartan potassium & $\mathrm{C}_{22} \mathrm{H}_{22} \mathrm{ClKN}_{6} \mathrm{O}$ & Ang II & An AT1 receptor antagonist \\
Telmisartan & $\mathrm{C}_{33} \mathrm{H}_{30} \mathrm{~N}_{4} \mathrm{O}_{2}$ & AT1 receptor & A long lasting antagonist of AT1 receptor \\
Methylprednisolone & $\mathrm{C}_{22} \mathrm{H}_{30} \mathrm{O}_{5}$ & Glucocorticoid receptor & Activate ACE2 and reduces IL-6 \\
TAPI-1 & $\mathrm{C}_{26} \mathrm{H}_{37} \mathrm{~N}_{5} \mathrm{O}_{5}$ & ADAM17 & Block the shedding of several cell surface proteins \\
Noscapine hydrochloride & $\mathrm{C}_{22} \mathrm{H}_{24} \mathrm{ClNO}_{7}$ & Bradykinin & a non-competitive Bradykinin inhibitor \\
SSR240612 & $\mathrm{C}_{42} \mathrm{H}_{53} \mathrm{ClN}_{4} \mathrm{O}_{7} \mathrm{~S}$ & B1R & A specific non-peptide bradykinin B1R antagonist \\
Icatibant acetate & $\mathrm{C}_{61} \mathrm{H}_{93} \mathrm{~N}_{19} \mathrm{O}_{15} \mathrm{~S}$ & B2R & A specific peptide antagonist of B2R \\
Fasitibant chloride & $\mathrm{C}_{36} \mathrm{H}_{49} \mathrm{Cl}_{3} \mathrm{~N}_{6} \mathrm{O}_{6} \mathrm{~S}$ & B2R & A selective nonpeptide bradykinin B2R antagonist & 177 \\
\end{tabular}

Table 5. Potential therapeutic tools for inhibiting HMGB1-RAGE/TLR4 signaling during COVID-19

\begin{tabular}{lllll}
\hline Potential therapeutic & Formula & Targets & Mechanism of action \\
\hline Glycyrrhizic acid & $\mathrm{C}_{42} \mathrm{H}_{62} \mathrm{O}_{16}$ & HMGB1 & A direct HMGB1 antagonist \\
Ammonium glycyrrhizinate & $\mathrm{C}_{42} \mathrm{H}_{65} \mathrm{NO}_{16}$ & $\mathrm{HMGB1}$ & A direct HMGB1 antagonist \\
FPS-ZM1 & $\mathrm{C}_{20} \mathrm{H}_{22} \mathrm{ClNO}_{18}$ & RAGE & A high-affinity RAGE inhibitor \\
Azeliragon & $\mathrm{C}_{32} \mathrm{H}_{38} \mathrm{ClN}_{3} \mathrm{O}_{2}$ & RAGE & An inhibitor of RAGE \\
\hline
\end{tabular}

Table 6. Potential therapeutic tools for antioxidant treatment during COVID-19

\begin{tabular}{lllll}
\hline Potential therapeutic & Formula & Targets & Mechanism of action \\
\hline Human recombinant IL-37 & & AMP-activated kinase & Increase NO bioavailability and reduces ROS formation \\
DAQ B1 & & Akt & An activator of Akt, and reduces oxidative stress \\
BMOV & & PTPase & An inhibitor of PTPase that activates eNOS and reduces oxidative stress ${ }^{187}$ \\
N-Acetyl-L-cysteine & $\mathrm{C}_{5} \mathrm{H}_{9} \mathrm{NO}_{3} \mathrm{~S}$ & Endogenous metabolite & A ROS inhibitor \\
VAS2870 & $\mathrm{C}_{18} \mathrm{H}_{12} \mathrm{~N}_{6} \mathrm{OS}$ & NOX & A pan NOX inhibitor & 185 \\
APX-115 & $\mathrm{C}_{17} \mathrm{H}_{18} \mathrm{ClN}_{3} \mathrm{O}$ & NOX & An active pan NOX inhibitor \\
Setanaxib & $\mathrm{C}_{21} \mathrm{H}_{19} \mathrm{CIN}_{4} \mathrm{O}_{2}$ & NOX1/4 & A selective NOX1/4 inhibitor \\
gp91ds-tat & $\mathrm{C}_{98} \mathrm{H}_{190} \mathrm{~N}_{50} \mathrm{O}_{22} \mathrm{~S}$ & NOX & Reduce ROS formation and platelet activation \\
GLX351322 & $\mathrm{C}_{21} \mathrm{H}_{25} \mathrm{~N}_{3} \mathrm{O}_{5} \mathrm{~S}$ & NOX4 & An inhibitor of NOX4 \\
GSK2795039 & $\mathrm{C}_{23} \mathrm{H}_{26} \mathrm{~N}_{6} \mathrm{O}_{2} \mathrm{~S}$ & NOX2 & A NOX2 inhibitor & 199 \\
\hline
\end{tabular}

Table 7. Potential therapeutic tools for targeting IL-6/IL-6R mediated signaling during COVID-19

\begin{tabular}{llll}
\hline Potential therapeutic & Formula & Targets & Mechanism of action \\
\hline Sarilumab & & IL-6 & A human immunoglobulin G1 monoclonal antibody. \\
Tocilizumab & & IL-6R & IL-6R neutralizing antibody \\
LMT-28 & $\mathrm{C}_{17} \mathrm{H}_{29} \mathrm{NO}_{4}$ & IL-6 & A synthetic IL-6 inhibitor that functions through direct binding to gp130 \\
Ruxolitinib & $\mathrm{C}_{17} \mathrm{H}_{18} \mathrm{~N}_{6}$ & JAK1/2 & A potent and selective JAK1/2 inhibitor \\
JAK-IN-1 & $\mathrm{C}_{20} \mathrm{H}_{24} \mathrm{~N}_{6} \mathrm{O}_{2}$ & JAK1/2/3 & A JAK1/2/3 inhibitor \\
JAK-IN-3 & $\mathrm{C}_{18} \mathrm{H}_{20} \mathrm{~N}_{4} \mathrm{O}_{3}$ & JAK1/3 & A potent JAK inhibitor \\
STAT3-IN-1 & $\mathrm{C}_{28} \mathrm{H}_{29} \mathrm{NO}_{6}$ & STAT3 & An excellent, selective and orally active STAT3 inhibitor \\
STAT3-IN-3 & $\mathrm{C}_{27} \mathrm{H}_{26} \mathrm{BrN}_{3} \mathrm{O}_{6} \mathrm{~S}$ & STAT3 & A potent and selective inhibitor of STAT3
\end{tabular}

kinase VEGF receptors (VEGFRs) in ECs to elicit various effects. ${ }^{116} \mathrm{~A}$ large body of evidence suggests that an increase in VEGF induces VEGFR2 activation through ERK1/2 and calcium signaling in ECs. ${ }^{116}$ VEGFA-stimulated VEGFR2 activation is an important process for modulating multiple biological responses, such as proliferation, survival, migration, and permeability. ${ }^{116}$ VEGFA/VEGFR2 recruits the TSAd adapter protein complex, which regulates VEGFAinduced activation of Src tyrosine kinase and vascular permeability in blood vascular ECs (Fig. 3). ${ }^{116}$ Notably, VEGFA is upregulated in the lungs of infections who have died from COVID-19. ${ }^{63}$ 
Endothelial activation and dysfunction in COVID-19: from basic mechanisms... Jin et al.

Table 8. Potential therapeutic tools for modulating complement activation, VEGFA/VEGFR2 Pathway, HSP90 and HIF-1 $\alpha$

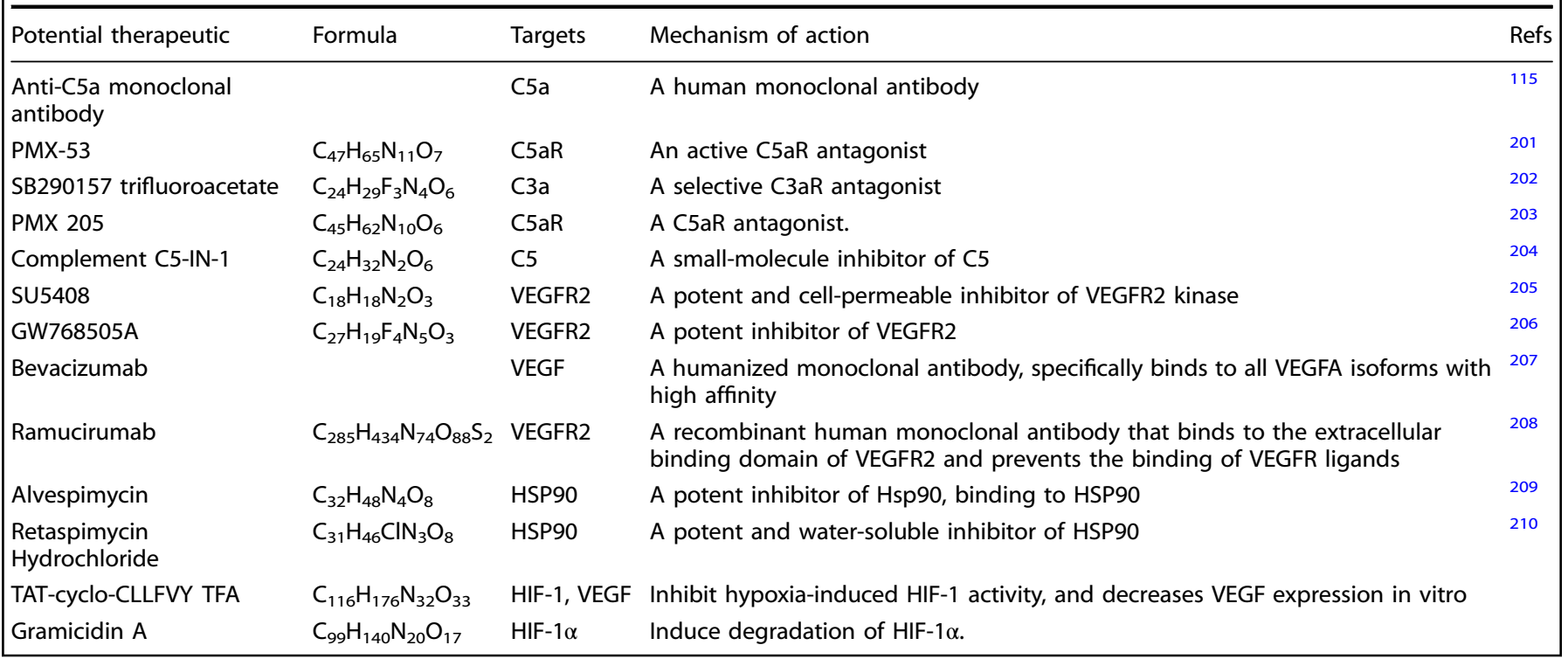

Collectively, targeting the VEGFA/VEGFR2 pathway is a possible therapeutic strategy for treatment of COVID-19. Potential therapeutic approaches for targeting VEGFA/VEGFR2 signaling are listed in Table 8.

CRP promotes endothelial activation and dysfunction

CRP is a major acute-phase protein, and pentamer CRP (pCRP) and monomer CRP (mCRP) are two of its subunits. Its circulating concentration is dramatically elevated at the onset of inflammation and infection. An increase in CRP is correlated with a poor prognosis of COVID-19. ${ }^{117,118}$ Recent studies have suggested that CRP plays a significant role in vascular inflammation and injury, which can damage ECs in vivo and in vitro. ${ }^{119} \mathrm{mCRP}$ can promote endothelial cell damage and apoptosis via the p38 pathway. ${ }^{120}$ CRP potently suppresses eNOS transcription in ECs and destabilizes eNOS mRNA, leading to endothelial dysfunction (Fig. 3). ${ }^{121}$ CRP has been demonstrated to upregulate adhesion molecules, facilitate EC apoptosis, and inhibit angiogenesis while augmenting CD14-induced endothelial activation. ${ }^{122,123}$ CRP also potently upregulates NF-KB, a key nuclear factor that can promote the transcription of inflammatory genes. ${ }^{123}$

Other pathways involved in endothelial activation and dysfunction Endothelial activation and dysfunction are caused by the combined actions of inflammatory mediators, leukocyte adhesion, and oxidants. In addition to the aforementioned mechanisms, heat shock protein (HSP) $90^{60}$ and hypoxia-inducible factor-1 (HIF-1) ${ }^{124}$ have also been suggested recently. HSPs belong to a group of highly conserved families of proteins expressed by all organisms, and their expression may be constitutive or inducible. HSPs are commonly considered protective molecules against different types of stress, such as oxidants, toxins, heavy metals, free radicals, and viruses. ${ }^{125}$ As mentioned earlier, a decline in the bioavailability of NO can cause endothelial dysfunction. ${ }^{23}$ The availability of NO to the vasculature is regulated by eNOS activity, and the involvement of HSP9O in the regulation of eNOS activity has been confirmed. ${ }^{126}$ The inhibition of HSP90 can prevent endothelial dysfunction. ${ }^{127}$ Fatal ARDS represented as hypoxia is the leading cause of death among COVID-19 patients. ${ }^{124}$ Local lung hypoxia is predicted to increase the transcription of HIF-1a, and in turn, HIF-1a signaling causes endothelial dysfunction. ${ }^{128}$ HIF-1a has been considered a target for treatment of COVID-19. ${ }^{124}$ Existing pharmacological modulators that act directly or indirectly on HSP90 and HIF-1a are listed in Table 8.

\section{CONCLUSIONS AND PERSPECTIVES}

The COVID-19 pandemic has posed an unprecedented challenge to the healthcare community. As our understanding of COVID-19 pathogenesis, endothelial activation and dysfunction are widely proposed by the international medical community. In this review, we summarized possible mechanisms of endothelial activation and dysfunction-mediated inflammation and abnormal coagulation based on clinical findings, suggesting that immunological and physiological functions of ECs, and multiple cellular signalingmediated endothelial activation and dysfunction should be given more attention. How this will inform specific anti-inflammatory treatments, thus far rather generically targeted, will be another field for proceeding investigation and innovation. Here, we have summarized the critical roles of ECs in the inflammatory process and detailed several mediators and signaling pathways in this cell type that contribute to inflammation. Recently, a lot of agents have been developed to control endothelial inflammation, usually with leukocytes and endothelial activation or dysfunction as the intended targets. The precise therapeutic mechanisms of the medications or monoclonal neutralizing antibodies recommended in this review should be confirmed in future clinical practice, and the efficacy of anticoagulants needs to be verified in welldesigned clinical trials. At present, a bulk of clinical and research data cannot be roughly interpreted.

To date, the pathogenesis of COVID-19 mostly remains unclear. The knowledge of the mechanisms of endothelial activation and dysfunction can be used to understand the pathogenesis of COVID-19. Uncontrolled inflammation is the common feature of severe COVID-19. Meanwhile, more attention should be paid to non-traditional forms of inflammation, as therapeutic tools will likely be extremely different for these pathways. For instance, endothelial inflammation has been rarely reported in the pathogenesis of many infectious diseases, but may be much more significant than we know. At last, as we present and interpret this evolving knowledge base, we need to find out which approaches to prevention and treatment of COVID-19, in this context, are most practicable and cost-effective. A collaborative effort between clinicians and biomedical investigators is urgently 
required to translate the present understanding of endotheliumpromoted inflammation to COVID-19 treatment.

\section{ACKNOWLEDGEMENTS}

The authors are supported by the National Science and Technology Specific Projects (NO.2018ZX10301407); the National Natural Science Foundation of China (NO.82002147 and NO.82073618); the Project founded by China Postdoctoral Science Foundation (NO.2019M662543).

\section{ADDITIONAL INFORMATION}

Competing interests: The authors declare no competing interests.

\section{REFERENCES}

1. Zhou, P. et al. A pneumonia outbreak associated with a new coronavirus of probable bat origin. Nature 579, 270-273 (2020).

2. Zhu, N. et al. A novel coronavirus from patients with pneumonia in China, 2019. N. Engl. J. Med. 382, 727-733 (2020).

3. World Health Organization. Coronavirus disease (COVID-2019) situation reports. Update on 24:00 of August 04.

4. Jin, Y. et al. Virology, epidemiology, pathogenesis, and control of COVID-19. Viruses 12, 372 (2020).

5. [The epidemiological characteristics of an outbreak of 2019 novel coronavirus diseases (COVID-19) in China]. Zhonghua liu xing bing. xue za zhi. 41, 145-151 (2020).

6. Huang, C. et al. Clinical features of patients infected with 2019 novel coronavirus in Wuhan, China. Lancet 395, 497-506 (2020).

7. Sturtzel, C. Endothelial cells. Adv. Exp. Med Biol. 1003, 71-91 (2017).

8. Zeng, $\mathrm{H}$. et al. Human pulmonary microvascular endothelial cells support productive replication of highly pathogenic avian influenza viruses: possible involvement in the pathogenesis of human H5N1 virus infection. J. Virol. 86, 667-678 (2012).

9. Aird, W. C. Phenotypic heterogeneity of the endothelium: II. Representative vascular beds. Circ. Res. 100, 174-190 (2007).

10. Maniatis, N. A. \& Orfanos, S. E. The endothelium in acute lung injury/acute respiratory distress syndrome. Curr. Opin. Crit. Care. 14, 22-30 (2008).

11. Matthay, M. A., McAuley, D. F. \& Ware, L. B. Clinical trials in acute respiratory distress syndrome: challenges and opportunities. Lancet Respir. Med. 5, 524-534 (2017).

12. Teuwen, L. A., Geldhof, V., Pasut, A. \& Carmeliet, P. COVID-19: the vasculature unleashed. Nat. Rev. Immunol. 20, 389-391 (2020).

13. Bermejo-Martin, J. F., Almansa, R., Torres, A., Gonzalez-Rivera, M. \& Kelvin, D. J. COVID-19 as a cardiovascular disease: the potential role of chronic endothelial dysfunction. Cardiovasc Res. 116, e132-e133 (2020).

14. Bermejo-Martin, J. F., Martin-Fernandez, M., Lopez-Mestanza, C., Duque, P. \& Almansa, R. Shared features of endothelial dysfunction between sepsis and Its preceding risk factors (aging and chronic disease). J. Clin. Med. 7, 400 (2018).

15. Nachman, R. L. \& Rafii, S. Platelets, petechiae, and preservation of the vascular wall. N. Engl. J. Med. 359, 1261-1270 (2008).

16. Iba, T. et al. The unique characteristics of COVID-19 coagulopathy. Crit. Care. 24, 360 (2020)

17. Levi, M. COVID-19 coagulopathy vs disseminated intravascular coagulation. Blood Adv. 4, 2850 (2020).

18. Klok, F. A. et al. Incidence of thrombotic complications in critically ill ICU patients with COVID-19. Thromb. Res. 191, 145-147 (2020).

19. Ciceri, F. et al. Microvascular COVID-19 lung vessels obstructive thromboinflammatory syndrome (MicroCLOTS): an atypical acute respiratory distress syndrome working hypothesis. Crit. Care Resusc. 22, 95-97 (2020).

20. Sinha, I. P. et al. Child poverty, food insecurity, and respiratory health during the COVID-19 pandemic. Lancet Respir. Med. 8, 762-763 (2020).

21. Sturtzel, C. in The Immunology of Cardiovascular Homeostasis and Pathology (eds Susanne Sattler \& Teresa Kennedy-Lydon) 71-91 (Springer International Publishing, 2017).

22. Godo, S. \& Shimokawa, H. Endothelial functions. Arterioscler Thromb. Vasc. Biol. 37, e108-e114 (2017).

23. Pober, J. S. \& Sessa, W. C. Evolving functions of endothelial cells in inflammation. Nat. Rev. Immunol. 7, 803-815 (2007).

24. Kruger-Genge, A., Blocki, A., Franke, R. P. \& Jung, F. Vascular endothelial cell biology: an update. Int. J. Mol. Sci. 20, 4411 (2019).

25. Tong, M. et al. Elevated serum endothelial cell adhesion molecules expression in COVID-19 patients. J. Infect. Dis. 222, 894-898 (2020).

26. Escher, R., Breakey, N. \& Lammle, B. Severe COVID-19 infection associated with endothelial activation. Thromb. Res. 190, 62 (2020).
27. Regev, T. et al. Pediatric inflammatory multisystem syndrome with central nervous system involvement and hypocomplementemia following Sars-Cov-2 Infection. Pediatr. Infect. Dis. J. 39, e206-e207 (2020).

28. Ouldali, N. et al. Emergence of Kawasaki disease related to SARS-CoV-2 infection in an epicentre of the French COVID-19 epidemic: a time-series analysis. Lancet Child Adolesc. Health 4, 662-668 (2020).

29. Green, S. J. Covid-19 accelerates endothelial dysfunction and nitric oxide deficiency. Microbes Infect. 22, 149-150 (2020).

30. Akerstrom, S., Gunalan, V., Keng, C. T., Tan, Y. J. \& Mirazimi, A. Dual effect of nitric oxide on SARS-CoV replication: viral RNA production and palmitoylation of the $S$ protein are affected. Virology 395, 1-9 (2009).

31. Akerstrom, $\mathrm{S}$. et al. Nitric oxide inhibits the replication cycle of severe acute respiratory syndrome coronavirus. J. Virol. 79, 1966-1969 (2005).

32. Reyes Gil, M. in Transfusion Medicine and Hemostasis (Third Edition) (eds Beth $\mathrm{H}$. Shaz, Christopher D. Hillyer, \& Morayma Reyes Gil) 559-564 (Elsevier, 2019).

33. Panigada, M. et al. Hypercoagulability of COVID-19 patients in intensive care unit: a report of thromboelastography findings and other parameters of hemostasis. J. Thromb. Haemost. 18, 1738-1742 (2020).

34. Ranucci, M. et al. The procoagulant pattern of patients with COVID-19 acute respiratory distress syndrome. J. Thromb. Haemost. 18, 1747-1751 (2020).

35. Figliozzi, S. et al. Predictors of adverse prognosis in Covid-19: a systematic review and meta-analysis. Eur. J. Clin. Invest. 50, e13362 (2020).

36. Iba, T., Levy, J. H., Levi, M., Connors, J. M. \& Thachil, J. Coagulopathy of coronavirus disease 2019. Crit. Care Med. 48, 1358-1364 (2020).

37. Liwinski, T., Zheng, D. \& Elinav, E. The microbiome and cytosolic innate immune receptors. Immunol. Rev. https://doi.org/10.1111/imr.12901 (2020).

38. Jin, Y., Zhang, R., Wu, W. \& Duan, G. Innate immunity evasion by enteroviruses linked to epidemic hand-foot-mouth disease. Front. Microbiol. 9, 2422 (2018).

39. de Wit, E., van Doremalen, N., Falzarano, D. \& Munster, V. J. SARS and MERS: recent insights into emerging coronaviruses. Nat. Rev. Microbiol. 14, 523-534 (2016).

40. Vabret, N. et al. Immunology of COVID-19: current state of the science. Immunity 52, 910-941 (2020).

41. Opitz, B., Eitel, J., Meixenberger, K. \& Suttorp, N. Role of Toll-like receptors, NODlike receptors and RIG-l-like receptors in endothelial cells and systemic infections. Thromb. Haemost. 102, 1103-1109 (2009).

42. Mai, J., Virtue, A., Shen, J., Wang, H. \& Yang, X. F. An evolving new paradigm: endothelial cells-conditional innate immune cells. J. Hematol. Oncol. 6, 61 (2013).

43. Tissari, J., Siren, J., Meri, S., Julkunen, I. \& Matikainen, S. IFN-alpha enhances TLR3mediated antiviral cytokine expression in human endothelial and epithelial cells by up-regulating TLR3 expression. J. Immunol. 174, 4289-4294 (2005).

44. El Kebir, D., Jozsef, L., Pan, W., Wang, L. \& Filep, J. G. Bacterial DNA activates endothelial cells and promotes neutrophil adherence through TLR9 signaling. J. Immunol. 182, 4386-4394 (2009).

45. Andonegui, G. et al. Endothelium-derived Toll-like receptor-4 is the key molecule in LPS-induced neutrophil sequestration into lungs. J. Clin. Invest. 111, 1011-1020 (2003).

46. Opitz, B. et al. Nod1-mediated endothelial cell activation by Chlamydophila pneumoniae. Circ. Res. 96, 319-326 (2005).

47. Opitz, B. et al. Listeria monocytogenes activated p38 MAPK and induced IL-8 secretion in a nucleotide-binding oligomerization domain 1-dependent manner in endothelial cells. J. Immunol. 176, 484-490 (2006).

48. Agostini, L. et al. NALP3 forms an IL-1beta-processing inflammasome with increased activity in Muckle-Wells autoinflammatory disorder. Immunity 20, 319-325 (2004).

49. Chen, H. et al. Cadmium induces NLRP3 inflammasome-dependent pyroptosis in vascular endothelial cells. Toxicol. Lett. 246, 7-16 (2016).

50. Xiang, M. et al. Hemorrhagic shock activation of NLRP3 inflammasome in lung endothelial cells. J. Immunol. 187, 4809-4817 (2011).

51. Freeman, T. L. \& Swartz, T. H. Targeting the NLRP3 Inflammasome in severe COVID-19. Front Immunol. 11, 1518 (2020).

52. Huang, J., Meyer, C. \& Zhu, C. T cell antigen recognition at the cell membrane. Mol. Immunol. 52, 155-164 (2012).

53. Goveia, J. et al. An integrated gene expression landscape profiling approach to identify lung tumor endothelial cell heterogeneity and angiogenic candidates. Cancer Cell. 37, 421 (2020).

54. Carman, C. V. \& Martinelli, R. in Encyclopedia of Cell Biology (eds Ralph A. Bradshaw \& Philip D. Stahl) 632-649 (Academic Press, 2016).

55. Hancock, W. W., Kraft, N. \& Atkins, R. C. The immunohistochemical demonstration of major histocompatibility antigens in the human kidney using monoclonal antibodies. Pathology 14, 409-414 (1982).

56. $\mathrm{Xu}, \mathrm{Z}$. et al. Pathological findings of COVID-19 associated with acute respiratory distress syndrome. Lancet Respir. Med. 8, 420-422 (2020).

57. Rao, R. M., Yang, L., Garcia-Cardena, G. \& Luscinskas, F. W. Endothelialdependent mechanisms of leukocyte recruitment to the vascular wall. Circ. Res. 101, 234-247 (2007). 
58. P-selectin. Rheumatology and Immunology Therapy. 735-736, (Springer Berlin Heidelberg, 2004).

59. Smadja, D. M. et al. Angiopoietin-2 as a marker of endothelial activation is a good predictor factor for intensive care unit admission of COVID-19 patients. Angiogenesis 23, 611-620 (2020).

60. Varga, Z. et al. Endothelial cell infection and endotheliitis in COVID-19. Lancet 395, 1417-1418 (2020).

61. Merrill, J. T., Erkan, D., Winakur, J. \& James, J. A. Emerging evidence of a COVID19 thrombotic syndrome has treatment implications. Nat. Rev. Rheumatol. 16, 581-589 (2020).

62. Divani, A. A. et al. Coronavirus disease 2019 and Stroke: clinical manifestations and pathophysiological insights. J. Stroke Cerebrovasc. Dis. 29, 104941 (2020).

63. Ackermann, M. et al. Pulmonary vascular endothelialitis, thrombosis, and angiogenesis in covid-19. N. Engl. J. Med. 383, 120-128 (2020).

64. Grobler, C. et al. Covid-19: the rollercoaster of fibrin(Ogen), D-Dimer, Von Willebrand factor, P-selectin and their interactions with endothelial cells, platelets and erythrocytes. Int. J. Mol. Sci. 21, 5168 (2020).

65. Nishiga, M., Wang, D. W., Han, Y., Lewis, D. B. \& Wu, J. C. COVID-19 and cardiovascular disease: from basic mechanisms to clinical perspectives. Nat. Rev. Cardiol. 17, 543-558 (2020).

66. Monteil, V. et al. Inhibition of SARS-CoV-2 infections in engineered human tissues using clinical-grade soluble human ACE2. Cell 181, 905-913 (2020).

67. Paniz-Mondolfi, A. et al. Central nervous system involvement by severe acute respiratory syndrome coronavirus-2 (SARS-CoV-2). J. Med Virol. 92, 699-702 (2020).

68. Su, H. et al. Renal histopathological analysis of 26 postmortem findings of patients with COVID-19 in China. Kidney Int. 98, 219-227 (2020).

69. Wang, C. et al. A human monoclonal antibody blocking SARS-CoV-2 infection. Nat. Commun. 11, 2251 (2020).

70. $\mathrm{Wu}, \mathrm{Y}$. et al. Identification of human single-domain antibodies against SARSCoV-2. Cell Host Microbe 27, 891-898 (2020).

71. Lovren, F. et al. Angiotensin converting enzyme-2 confers endothelial protection and attenuates atherosclerosis. Am. J. Physiol. Heart Circ. Physiol. 295, H1377-H1384 (2008).

72. Kuba, K. et al. A crucial role of angiotensin converting enzyme 2 (ACE2) in SARS coronavirus-induced lung injury. Nat. Med. 11, 875-879 (2005).

73. Eguchi, S., Kawai, T., Scalia, R. \& Rizzo, V. Understanding angiotensin II type 1 receptor signaling in vascular pathophysiology. Hypertension 71, 804-810 (2018).

74. Haga, S. et al. Modulation of TNF-alpha-converting enzyme by the spike protein of SARS-CoV and ACE2 induces TNF-alpha production and facilitates viral entry. Proc. Natl Acad. Sci. USA 105, 7809-7814 (2008)

75. Sriram, K. \& Insel, P. A. A hypothesis for pathobiology and treatment of COVID19: The centrality of ACE1/ACE2 imbalance. Br. J. Pharmacol. 177, 4825-4844 (2020).

76. Liu, Y. et al. Clinical and biochemical indexes from 2019-nCoV infected patients linked to viral loads and lung injury. Sci. China Life Sci. 63, 364-374 (2020).

77. Lobov, I. B., Brooks, P. C. \& Lang, R. A. Angiopoietin-2 displays VEGF-dependent modulation of capillary structure and endothelial cell survival in vivo. Proc. Natl Acad. Sci. USA 99, 11205-11210 (2002).

78. Akwii, R. G., Sajib, M. S., Zahra, F. T. \& Mikelis, C. M. Role of angiopoietin-2 in Vascular physiology and pathophysiology. Cells 8, 471 (2019).

79. Scholz, A., Plate, K. H. \& Reiss, Y. Angiopoietin-2: a multifaceted cytokine that functions in both angiogenesis and inflammation. Ann. N. Y Acad. Sci. 1347 45-51 (2015).

80. Didion, S. P. Cellular and oxidative mechanisms associated with interleukin- 6 signaling in the vasculature. Int J. Mol. Sci. 18, 2563 (2017).

81. De Mello, W. C. Chemical communication between heart cells is disrupted by intracellular renin and angiotensin II: implications for heart development and disease. Front. Endocrinol. 6, 72 (2015).

82. Geranmayeh, M. H., Rahbarghazi, R. \& Farhoudi, M. Targeting pericytes for neurovascular regeneration. Cell Commun. Signal. 17, 26 (2019).

83. Fagiani, E. \& Christofori, G. Angiopoietins in angiogenesis. Cancer Lett. 328 18-26 (2013).

84. Bossi, F., Bulla, R. \& Tedesco, F. Endothelial cells are a target of both complement and kinin system. Int Immunopharmacol. 8, 143-147 (2008).

85. Mombouli, J. V. \& Vanhoutte, P. M. Kinins and endothelial control of vascular smooth muscle. Annu Rev. Pharm. Toxicol. 35, 679-705 (1995).

86. van de Veerdonk, F. L. et al. Kallikrein-kinin blockade in patients with COVID-19 to prevent acute respiratory distress syndrome. Elife 9, e57555 (2020).

87. Sodhi, C. P. et al. Attenuation of pulmonary ACE2 activity impairs inactivation of des-Arg(9) bradykinin/BKB1R axis and facilitates LPS-induced neutrophil infiltration. Am. J. Physiol. Lung Cell Mol. Physiol. 314, L17-L31 (2018).

88. Figueroa, C. D. et al. Kinin B1 receptor regulates interactions between neutrophils and endothelial cells by modulating the levels of Mac-1, LFA-1 and intercellular adhesion molecule-1. Innate Immun. 21, 289-304 (2015).
89. Lopatko Fagerstrom, I. et al. Blockade of the kallikrein-kinin system reduces endothelial complement activation in vascular inflammation. EBioMedicine $\mathbf{4 7}$ 319-328 (2019).

90. Asavarut, P., Zhao, H., Gu, J. \& Ma, D. The role of HMGB1 in inflammationmediated organ injury. Acta Anaesthesiol. Taiwan. 51, 28-33 (2013).

91. Wolfson, R. K., Chiang, E. T. \& Garcia, J. G. HMGB1 induces human lung endothelial cell cytoskeletal rearrangement and barrier disruption. Microvasc. Res. 81, 189-197 (2011).

92. Yang, $H$. et al. A critical cysteine is required for HMGB1 binding to Toll-like receptor 4 and activation of macrophage cytokine release. Proc. Natl Acad. Sci. USA 107, 11942-11947 (2010).

93. Yang, H. et al. MD-2 is required for disulfide HMGB1-dependent TLR4 signaling. J. Exp. Med. 212, 5-14 (2015).

94. Luo, Y., Li, S. J., Yang, J., Qiu, Y. Z. \& Chen, F. P. HMGB1 induces an inflammatory response in endothelial cells via the RAGE-dependent endoplasmic reticulum stress pathway. Biochem Biophys. Res Commun. 438, 732-738 (2013).

95. Huang, W. et al. HMGB1 increases permeability of the endothelial cell monolayer via RAGE and Src Family tyrosine kinase pathways. Inflammation 35, 350-362 (2012).

96. Huang, W. et al. High-mobility group box 1 impairs airway epithelial barrier function through the activation of the RAGE/ERK pathway. Int J. Mol. Med. 37, 1189-1198 (2016).

97. Qu, L. et al. High-mobility group Box 1 (HMGB1) and autophagy in acute lung injury (ALI): a review. Med Sci. Monit. 25, 1828-1837 (2019).

98. Simpson, J. et al. Respiratory syncytial virus infection promotes necroptosis and HMGB1 release by airway epithelial cells. Am. J. Respir. Crit. Care Med. 201, 1358-1371 (2020).

99. Chen, G. et al. Pathogenic role of HMGB1 in SARS? Med Hypotheses 63, 691-695 (2004).

100. Andersson, U., Ottestad, W. \& Tracey, K. J. Extracellular HMGB1: a therapeutic target in severe pulmonary inflammation including COVID-19? Mol. Med. 26, 42 (2020).

101. Pennathur, S. \& Heinecke, J. W. Oxidative stress and endothelial dysfunction in vascular disease. Curr. Diabetes Rep. 7, 257-264 (2007).

102. Konior, A., Schramm, A., Czesnikiewicz-Guzik, M. \& Guzik, T. J. NADPH oxidases in vascular pathology. Antioxid. Redox Signal. 20, 2794-2814 (2014).

103. Di, A., Mehta, D. \& Malik, A. B. ROS-activated calcium signaling mechanisms regulating endothelial barrier function. Cell Calcium 60, 163-171 (2016).

104. Scioli, M. G. et al. Oxidative stress and new pathogenetic mechanisms in endothelial dysfunction: potential diagnostic biomarkers and therapeutic targets. J. Clin. Med. 9, 1995 (2020).

105. Violi, F. et al. Nox2 activation in covid-19. Redox Biol. 36, 101655 (2020).

106. Liu, F. et al. Clinically significant portal hypertension in cirrhosis patients with COVID-19: clinical characteristics and outcomes. J. Infect. 81, e178-e180 (2020).

107. Ataie-Kachoie, P., Pourgholami, M. H., Richardson, D. R. \& Morris, D. L. Gene of the month: interleukin 6 (IL-6). J. Clin. Pathol. 67, 932-937 (2014).

108. Merle, N. S., Noe, R., Halbwachs-Mecarelli, L., Fremeaux-Bacchi, V. \& Roumenina, L. T. Complement system part II: role in immunity. Front Immunol. 6, 257 (2015).

109. Walport, M. J. Complement. N. Engl. J. Med. 344, 1058-1066 (2001).

110. Maglakelidze, N., Manto, K. M. \& Craig, T. J. A review: does complement or the contact system have a role in protection or pathogenesis of COVID-19? Pulm. Ther. 6, 169-176 (2020).

111. Noris, M., Benigni, A. \& Remuzzi, G. The case of complement activation in COVID19 multiorgan impact. Kidney Int. 98, 314-322 (2020).

112. Cugno, M. et al. Complement activation in patients with COVID-19: A novel therapeutic target. J. Allergy Clin. Immunol. 146, 215-217 (2020).

113. Ricklin, D., Reis, E. S. \& Lambris, J. D. Complement in disease: a defence system turning offensive. Nat. Rev. Nephrol. 12, 383-401 (2016).

114. Kawana, S. The membrane attack complex of complement alters the membrane integrity of cultured endothelial cells: a possible pathophysiology for immune complex vasculitis. Acta Derm. Venereol. 76, 13-16 (1996).

115. Giudice, V. et al. Combination of ruxolitinib and eculizumab for treatment of severe SARS-CoV-2-related acute respiratory distress syndrome: a controlled study. Front Pharmacol. 11, 857 (2020).

116. Karaman, S., Leppanen, V. M. \& Alitalo, K. Vascular endothelial growth factor signaling in development and disease. Development 145, dev151019 (2018).

117. Wang, L. C-reactive protein levels in the early stage of COVID-19. Med Mal. Infect. 50, 332-334 (2020)

118. Luo, X. et al. Prognostic value of C-reactive protein in patients with COVID-19. Clin. Infect. Dis. 71, 2174-2179 (2020).

119. Devaraj, S., Kumaresan, P. R. \& Jialal, I. C-reactive protein induces release of both endothelial microparticles and circulating endothelial cells in vitro and in vivo: further evidence of endothelial dysfunction. Clin. Chem. 57, 1757-1761 (2011).

120. Melnikov, I. S. et al. Current position on the role of monomeric C-reactive protein in vascular pathology and atherothrombosis. Curr. Pharm. Des. 26, 37-43 (2020).

121. Verma, S. et al. A self-fulfilling prophecy: C-reactive protein attenuates nitric oxide production and inhibits angiogenesis. Circulation 106, 913-919 (2002). 
122. Verma, S. et al. Endothelin antagonism and interleukin- 6 inhibition attenuate the proatherogenic effects of C-reactive protein. Circulation 105, 1890-1896 (2002).

123. Szmitko, P. E. et al. New markers of inflammation and endothelial cell activation: Part I. Circulation 108, 1917-1923 (2003).

124. Afsar, B., Kanbay, M. \& Afsar, R. E. Hypoxia inducible factor-1 protects against COVID-19: A hypothesis. Med Hypotheses 143, 109857 (2020).

125. Shankar, K. \& Mehendale, H. M. Heat-Shock Proteins. Encyclopedia of Toxicology (Third Edition), 830-831 (Academic Press, 2014).

126. Mohan, S., Konopinski, R., Yan, B., Centonze, V. E. \& Natarajan, M. High glucoseinduced IKK-Hsp-90 interaction contributes to endothelial dysfunction. Am. J. Physiol. Cell Physiol. 296, C182-C192 (2009).

127. Joshi, A. D. et al. Heat shock protein 90 inhibitors prevent LPS-induced endothelial barrier dysfunction by disrupting RhoA signaling. Am. J. Respir. Cell Mol. Biol. 50, 170-179 (2014).

128. $\mathrm{Xi}$, Y. et al. Local lung hypoxia determines epithelial fate decisions during alveolar regeneration. Nat. Cell Biol. 19, 904-914 (2017).

129. Franklin, B. S. et al. Therapeutical targeting of nucleic acid-sensing Toll-like receptors prevents experimental cerebral malaria. Proc. Natl Acad. Sci. USA 108, 3689-3694 (2011).

130. Iwata, Y. et al. p38 Mitogen-activated protein kinase contributes to autoimmune renal injury in MRL-Fas Ipr mice. J. Am. Soc. Nephrol. 14, 57-67 (2003).

131. Kecek Plesec, K. et al. Identification of indole scaffold-based dual inhibitors of NOD1 and NOD2. Bioorg. Med Chem. 24, 5221-5234 (2016).

132. Hershberger, P. M. et al. Synthesis and physicochemical characterization of novel phenotypic probes targeting the nuclear factor-kappa B signaling pathway. Beilstein J. Org. Chem. 9, 900-907 (2013).

133. Rickard, D. J. et al. Identification of benzimidazole diamides as selective inhibitors of the nucleotide-binding oligomerization domain 2 (NOD2) signaling pathway. PloS one 8, e69619 (2013).

134. Du, Y. et al. Muscone improves cardiac function in mice after myocardial infarction by alleviating cardiac macrophage-mediated chronic inflammation through inhibition of NF-kappaB and NLRP3 inflammasome. Am. J. Transl. Res. 10, 4235-4246 (2018).

135. Jiang, $H$. et al. Identification of a selective and direct NLRP3 inhibitor to treat inflammatory disorders. J. Exp. Med. 214, 3219-3238 (2017).

136. Cocco, M. et al. Development of an acrylate derivative targeting the NLRP3 inflammasome for the treatment of inflammatory bowel disease. J. Med Chem. 60, 3656-3671 (2017).

137. Hachmo, Y. et al. The small tellurium compound AS101 ameliorates rat crescentic glomerulonephritis: association with inhibition of macrophage caspase-1 activity via very late antigen-4 inactivation. Front Immunol. 8, 240 (2017).

138. Wang, C. P. et al. Mulberroside A protects against ischemic impairment in primary culture of rat cortical neurons after oxygen-glucose deprivation followed by reperfusion. J. Neurosci. Res. 92, 944-954 (2014).

139. Ajayi, F. O., Salako, L. A. \& Kuye, J. O. Comparison of the partitioning in vitro of chloroquine and its desethyl metabolites between the erythrocytes and plasma of healthy subjects and those with falciparum malaria. Afr. J. Med Med Sci. 18, 95-100 (1989).

140. Zhou, K. et al. Schaftoside ameliorates oxygen glucose deprivation-induced inflammation associated with the TLR4/Myd88/Drp1-related mitochondrial fission in BV2 microglia cells. J. Pharm. Sci. 139, 15-22 (2019).

141. Ono, Y. et al. TAK-242, a specific inhibitor of Toll-like receptor 4 signalling, prevents endotoxemia-induced skeletal muscle wasting in mice. Sci. Rep. 10, 694 (2020)

142. Nizamutdinova, I. T. et al. Hesperidin, hesperidin methyl chalone and phellopterin from Poncirus trifoliata (Rutaceae) differentially regulate the expression of adhesion molecules in tumor necrosis factor-alpha-stimulated human umbilical vein endothelial cells. Int Immunopharmacol. 8, 670-678 (2008).

143. Kikuchi, J. et al. The novel orally active proteasome inhibitor K-7174 exerts antimyeloma activity in vitro and in vivo by down-regulating the expression of class I histone deacetylases. J. Biol. Chem. 288, 25593-25602 (2013).

144. Shimada, T. et al. Unexpected blockade of adipocyte differentiation by K-7174: implication for endoplasmic reticulum stress. Biochem Biophys. Res Commun. 363, 355-360 (2007).

145. Imagawa, S. et al. A GATA-specific inhibitor (K-7174) rescues anemia induced by IL-1beta, TNF-alpha, or L-NMMA. FASEB J. 17, 1742-1744 (2003).

146. Hutchinson, $M$. Natalizumab: a new treatment for relapsing remitting multiple sclerosis. Ther. Clin. Risk Manag. 3, 259-268 (2007).

147. Huang, T. H., Tran, V. H., Roufogalis, B. D. \& Li, Y. Gypenoside XLIX, a naturally occurring PPAR-alpha activator, inhibits cytokine-induced vascular cell adhesion molecule-1 expression and activity in human endothelial cells. Eur. J. Pharmacol. 565, 158-165 (2007)

148. Zhu, G. D. et al. Selective inhibition of ICAM-1 and E-selectin expression in human endothelial cells. 2. Aryl modifications of 4-(aryloxy)thieno[2,3-c]pyridines with fine-tuning at C-2 carbamides. J. Med Chem. 44, 3469-3487 (2001).
149. Morita, K. et al. RUNX transcription factors potentially control E-selectin expression in the bone marrow vascular niche in mice. Blood Adv. 2, 509-515 (2018).

150. Rao, V. R. et al. Delivery of SAR 1118 to the retina via ophthalmic drops and its effectiveness in a rat streptozotocin (STZ) model of diabetic retinopathy (DR). Invest Ophthalmol. Vis. Sci. 51, 5198-5204 (2010).

151. Sun, Y., Zhang, R., Gadek, T. R., O'Neill, C. A. \& Pearlman, E. Corneal inflammation is inhibited by the LFA-1 antagonist, lifitegrast (SAR 1118). J. Ocul. Pharm. Ther. 29, 395-402 (2013).

152. Sanfilippo, P. J. et al. Novel thiazole based heterocycles as inhibitors of LFA-1/ ICAM-1 mediated cell adhesion. J. Med Chem. 38, 1057-1059 (1995).

153. Watterson, S. H. et al. Small molecule antagonist of leukocyte function associated antigen-1 (LFA-1): structure-activity relationships leading to the identification of 6-((5S,9R)-9-(4-cyanophenyl)-3-(3,5-dichlorophenyl)-1-methyl-2,4dioxo-1,3,7-tria zaspiro[4.4]nonan-7-yl)nicotinic acid (BMS-688521). J. Med Chem. 53, 3814-3830 (2010).

154. Palma-Vargas, J. M. et al. Small-molecule selectin inhibitor protects against liver inflammatory response after ischemia and reperfusion. J. Am. Coll. Surg. 185, 365-372 (1997).

155. Xia, Y. F. et al. Andrographolide attenuates inflammation by inhibition of NFkappa B activation through covalent modification of reduced cysteine 62 of p50. J. Immunol. 173, 4207-4217 (2004).

156. Lasky, L. A. Selectins: interpreters of cell-specific carbohydrate information during inflammation. Science 258, 964-969 (1992).

157. Myers, D. D. Jr. et al. Resolution of venous thrombosis using a novel oral smallmolecule inhibitor of P-selectin (PSI-697) without anticoagulation. Thromb. Haemost. 97, 400-407 (2007).

158. Aisiku, O. et al. Parmodulins inhibit thrombus formation without inducing endothelial injury caused by vorapaxar. Blood 125, 1976-1985 (2015).

159. Gandhi, D. M. et al. Characterization of Protease-Activated Receptor (PAR) ligands: Parmodulins are reversible allosteric inhibitors of PAR1-driven calcium mobilization in endothelial cells. Bioorg. Med Chem. 26, 2514-2529 (2018).

160. Thachil, J. et al. ISTH interim guidance on recognition and management of coagulopathy in COVID-19. J. Thromb. Haemost. 18, 1023-1026 (2020).

161. Tang, N. et al. Anticoagulant treatment is associated with decreased mortality in severe coronavirus disease 2019 patients with coagulopathy. J. Thromb. Haemost. 18, 1094-1099 (2020)

162. Shin, H. S. Empirical treatment and prevention of COVID-19. Infect. Chemother. 52, 142-153 (2020).

163. Glas, G. J. et al. Bronchoalveolar hemostasis in lung injury and acute respiratory distress syndrome. J. Thromb. Haemost. 11, 17-25 (2013).

164. Moore, H. B. et al. Is there a role for tissue plasminogen activator as a novel treatment for refractory COVID-19 associated acute respiratory distress syndrome? J. Trauma Acute Care Surg. 88, 713-714 (2020).

165. Ahrens, I., Lip, G. Y. \& Peter, K. New oral anticoagulant drugs in cardiovascular disease. Thromb. Haemost. 104, 49-60 (2010).

166. Xiang, Z. et al. Glucocorticoids improve severe or critical COVID-19 by activating ACE2 and reducing IL-6 levels. Int J. Biol. Sci. 16, 2382-2391 (2020).

167. Hobl, E. L. et al. Reversal strategy in antagonizing the P2Y12 -inhibitor ticagrelor. Eur. J. Clin. Invest. 43, 1258-1261 (2013).

168. Aungraheeta, R. et al. Inverse agonism at the P2Y12 receptor and ENT1 transporter blockade contribute to platelet inhibition by ticagrelor. Blood $\mathbf{1 2 8}$, 2717-2728 (2016).

169. Sugidachi, A. et al. The greater in vivo antiplatelet effects of prasugrel as compared to clopidogrel reflect more efficient generation of its active metabolite with similar antiplatelet activity to that of clopidogrel's active metabolite. J. Thromb. Haemost. 5, 1545-1551 (2007).

170. Angiolillo, D. J. et al. Pharmacokinetic and pharmacodynamic effects of elinogrel: results of the platelet function substudy from the intravenous and oral administration of elinogrel to evaluate tolerability and efficacy in nonurgent percutaneous coronary intervention patients (INNOVATE-PCI) trial. Circ. Cardiovasc Interv. 5, 347-356 (2012)

171. Hernandez Prada, J. A. et al. Structure-based identification of small-molecule angiotensin-converting enzyme 2 activators as novel antihypertensive agents. Hypertension 51, 1312-1317 (2008).

172. Soldner, A., Spahn-Langguth, H., Palm, D. \& Mutschler, E. A radioreceptor assay for the analysis of AT1-receptor antagonists. Correlation with complementary LC data reveals a potential contribution of active metabolites. J. Pharm. Biomed. Anal. 17, 111-124 (1998).

173. Chen, Y. et al. Lipoxin A4 and its analogue suppress the tumor growth of transplanted $\mathrm{H} 22$ in mice: the role of antiangiogenesis. Mol. Cancer Ther. 9, 2164-2174 (2010).

174. Habashi, J. P. et al. Losartan, an AT1 antagonist, prevents aortic aneurysm in a mouse model of Marfan syndrome. Science 312, 117-121 (2006). 
175. Torika, N., Asraf, K., Cohen, H. \& Fleisher-Berkovich, S. Intranasal telmisartan ameliorates brain pathology in five familial Alzheimer's disease mice. Brain Behav. Immun. 64, 80-90 (2017).

176. Moss, M. L. \& Minond, D. Recent advances in ADAM17 research: a promising target for cancer and inflammation. Mediators Inflamm. 2017, 9673537 (2017).

177. Lokhorst, B., Rolfes, L. \& Jessurun, N. T. Interaction of OTC drug noscapine and acenocoumarol and phenprocoumon. Br. J. Clin. Pharmacol. 85, 1041-1043 (2019).

178. Dias, J. P. et al. The kinin B1 receptor antagonist SSR240612 reverses tactile and cold allodynia in an experimental rat model of insulin resistance. Br. J. Pharmacol. 152, 280-287 (2007).

179. Bawolak, M. T., Fortin, J. P., Vogel, L. K., Adam, A. \& Marceau, F. The bradykinin B2 receptor antagonist icatibant (Hoe 140) blocks aminopeptidase $\mathrm{N}$ at micromolar concentrations: off-target alterations of signaling mediated by the bradykinin B1 and angiotensin receptors. Eur. J. Pharmacol. 551, 108-111 (2006).

180. Valenti, C., Giuliani, S., Cialdai, C., Tramontana, M. \& Maggi, C. A. Fasitibant chloride, a kinin $B(2)$ receptor antagonist, and dexamethasone interact to inhibit carrageenaninduced inflammatory arthritis in rats. Br. J. Pharmacol. 166, 1403-1410 (2012).

181. Wang, L. Y. et al. Glycyrrhizic acid increases glucagon like peptide-1 secretion via TGR5 activation in type 1-like diabetic rats. Biomed. Pharmacother. 95, 599-604 (2017).

182. Li, C. et al. Glycyrrhizin, a direct HMGB1 antagonist, ameliorates inflammatory infiltration in a model of autoimmune thyroiditis via inhibition of TLR2-HMGB1 Signaling. Thyroid 27, 722-731 (2017).

183. Deane, R. et al. A multimodal RAGE-specific inhibitor reduces amyloid betamediated brain disorder in a mouse model of Alzheimer disease. J. Clin. Invest. 122, 1377-1392 (2012).

184. Chen, Y. et al. RAGE ligation affects $T$ cell activation and controls $T$ cell differentiation. J. Immunol. 181, 4272-4278 (2008).

185. Ballak, D. B. et al. Short-term interleukin-37 treatment improves vascular endothelial function, endurance exercise capacity, and whole-body glucose metabolism in old mice. Aging Cell. 19, e13074 (2020).

186. Shah, D. I. \& Singh, M. Effect of demethylasterriquinone b1 in hypertension associated vascular endothelial dysfunction. Int J. Cardiol. 120, 317-324 (2007).

187. Shah, D. I. \& Singh, M. Inhibition of protein tyrosin phosphatase improves vascular endothelial dysfunction. Vasc. Pharmacol. 44, 177-182 (2006).

188. Halasi, M. et al. ROS inhibitor N-acetyl-L-cysteine antagonizes the activity of proteasome inhibitors. Biochem J. 454, 201-208 (2013).

189. Sancho, P. \& Fabregat, I. The NADPH oxidase inhibitor VAS2870 impairs cell growth and enhances TGF-beta-induced apoptosis of liver tumor cells. Biochem Pharmacol. 81, 917-924 (2011).

190. Cha, J. J. et al. APX-115, a first-in-class pan-NADPH oxidase (Nox) inhibitor, protects db/db mice from renal injury. Lab Invest. 97, 419-431 (2017).

191. Green, D. E. et al. The Nox4 inhibitor GKT137831 attenuates hypoxia-induced pulmonary vascular cell proliferation. Am. J. Respir. Cell Mol. Biol. 47, 718-726 (2012)

192. Lopes, N. et al. Thrombospondin 2 regulates cell proliferation induced by Rac1 redox-dependent signaling. Mol. Cell Biol. 23, 5401-5408 (2003).

193. Anvari, E., Wikstrom, P., Walum, E. \& Welsh, N. The novel NADPH oxidase 4 inhibitor GLX351322 counteracts glucose intolerance in high-fat diet-treated C57BL/6 mice. Free Radic. Res. 49, 1308-1318 (2015).

194. Hirano, K. et al. Discovery of GSK2795039, a novel small molecule NADPH oxidase 2 inhibitor. Antioxid. Redox Signal. 23, 358-374 (2015).

195. Xu, C., Su, Y., Paccaly, A. \& Kanamaluru, V. Population pharmacokinetics of sarilumab in patients with rheumatoid arthritis. Clin. Pharmacokinet. 58, 1455-1467 (2019).
196. Zhang, C., Wu, Z., Li, J. W., Zhao, H. \& Wang, G. Q. Cytokine release syndrome in severe COVID-19: interleukin-6 receptor antagonist tocilizumab may be the key to reduce mortality. Int J. Antimicrob. Agents 55, 105954 (2020).

197. Hong, S. S. et al. A novel small-molecule inhibitor targeting the IL-6 receptor beta subunit, glycoprotein 130. J. Immunol. 195, 237-245 (2015).

198. Soth, M. et al. 3-Amido pyrrolopyrazine JAK kinase inhibitors: development of a JAK3 vs JAK1 selective inhibitor and evaluation in cellular and in vivo models. J. Med Chem. 56, 345-356 (2013).

199. Spergel, S. H. et al. Discovery of a JAK1/3 inhibitor and use of a prodrug to demonstrate efficacy in a model of rheumatoid arthritis. ACS Med Chem. Lett. 10, 306-311 (2019).

200. Li, S. et al. Discovery of oral-available resveratrol-caffeic acid based hybrids inhibiting acetylated and phosphorylated STAT3 protein. Eur. J. Med Chem. 124 1006-1018 (2016)

201. Finch, A. M. et al. Low-molecular-weight peptidic and cyclic antagonists of the receptor for the complement factor C5a. J. Med Chem. 42, 1965-1974 (1999).

202. Hutamekalin, P. et al. Effect of the C3a-receptor antagonist SB 290157 on antiOVA polyclonal antibody-induced arthritis. J. Pharm. Sci. 112, 56-63 (2010).

203. Woodruff, T. M. et al. The complement factor $\mathrm{C} 5 \mathrm{a}$ contributes to pathology in a rat model of amyotrophic lateral sclerosis. J. Immunol. 181, 8727-8734 (2008).

204. Jendza, K. et al. A small-molecule inhibitor of C5 complement protein. Nat Chem. Biol. 15, 666-668 (2019).

205. Sun, L. et al. Synthesis and biological evaluations of 3-substituted indolin-2ones: a novel class of tyrosine kinase inhibitors that exhibit selectivity toward particular receptor tyrosine kinases. J. Med Chem. 41, 2588-2603 (1998).

206. Elkins, J. M. et al. Comprehensive characterization of the Published Kinase Inhibitor Set. Nat. Biotechnol. 34, 95-103 (2016).

207. Di Mauro, C. et al. Hedgehog signalling pathway orchestrates angiogenesis in triple-negative breast cancers. Br. J. Cancer 116, 1425-1435 (2017).

208. Fala, L. Cyramza (Ramucirumab) Approved for the Treatment of Advanced Gastric Cancer and Metastatic Non-Small-Cell Lung Cancer. Am. Health Drug Benefits 8, 49-53 (2015).

209. Henke, A. et al. Reduced contractility and motility of prostatic cancer-associated fibroblasts after inhibition of heat shock protein 90. Cancers (Basel). 8, 77 (2016).

210. Scaltriti, M. et al. Antitumor activity of the Hsp90 inhibitor IPI-504 in HER2positive trastuzumab-resistant breast cancer. Mol. Cancer Ther. 10, 817-824 (2011).

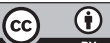

Open Access This article is licensed under a Creative Commons Attribution 4.0 International License, which permits use, sharing adaptation, distribution and reproduction in any medium or format, as long as you give appropriate credit to the original author(s) and the source, provide a link to the Creative Commons license, and indicate if changes were made. The images or other third party material in this article are included in the article's Creative Commons license, unless indicated otherwise in a credit line to the material. If material is not included in the article's Creative Commons license and your intended use is not permitted by statutory regulation or exceeds the permitted use, you will need to obtain permission directly from the copyright holder. To view a copy of this license, visit http://creativecommons. org/licenses/by/4.0/.

(c) The Author(s) 2021 